\title{
A Comprehensive Overview and Characterization of Wireless Channels for Networked Robotic and Control Systems
}

\author{
Alejandro Gonzalez-Ruiz, Alireza Ghaffarkhah, and Yasamin Mostofi \\ Department of Electrical and Computer Engineering, University of New Mexico, Albuquerque, NM 87131, USA \\ Correspondence should be addressed to Alireza Ghaffarkhah, alinem@ece.unm.edu
}

Received 25 July 2011; Accepted 11 October 2011

Academic Editor: Heinz Wörn

Copyright ( $) 2011$ Alejandro Gonzalez-Ruiz et al. This is an open access article distributed under the Creative Commons Attribution License, which permits unrestricted use, distribution, and reproduction in any medium, provided the original work is properly cited.

\begin{abstract}
The goal of this overview paper is to serve as a reference for researchers that are interested in the realistic modeling of wireless channels for the purpose of analysis and optimization of networked robotic systems. By utilizing the knowledge available in the wireless communication literature, we first summarize a probabilistic framework for the characterization of the underlying multiscale dynamics of a wireless link. We furthermore confirm this framework with our robotic testbed, by making an extensive number of channel measurements. To show the usefulness of this framework for networked robotic applications, we then discuss a few recent examples where this probabilistic channel characterization has been utilized for the theoretical analysis and communication-aware design of networked robotic systems. Finally, we show how to develop a realistic yet simple channel simulator, which can be used to verify cooperative robotic operations in the presence of realistic communication links.
\end{abstract}

\section{Introduction}

Recently, there has been considerable interest in multi-agent robotic networks. In order to realize the full potential of such systems, an integrative approach to communication and robotic issues is essential. In the robotics community, progress has been made towards designing local decisions that can result in certain group behaviors [1-7]. Similarly, in the communications community, rich literature was developed, over the past decades, for the characterization and modeling of wireless channels. However, the knowledge available on wireless link characterization is not typically used in networked robotic/control literature, that is, ideal or oversimplified models are commonly used. It is, therefore, the goal of this paper to provide a reference for the characterization and modeling of wireless channels for the analysis and optimization of networked robotic systems.

In a realistic communication setting, such as an urban area or an indoor environment, Line-Of-Sight (LOS) communication may not be possible due to the existence of several objects that can attenuate, reflect, diffract, or block the transmitted signal. The received signal power typically experiences considerable variations and can change drastically in even a small distance. As an example, consider Figure 1, where channel measurements in the Electrical and Computer Engineering (ECE) building at UNM are shown. It can be seen that channel can change drastically with a small movement of the robot. Thus, communication between robotic units can degrade due to factors such as shadowing, fading, or distance-dependent path loss [8], which can impact the overall performance of the robotic network considerably. However, multiagent robotic and navigation literature typically consider ideal links or links that are ideal within a certain radius of the node since distributed and cooperative control of robotic units is already a considerably challenging problem. Such simplified link models, however, do not embrace realistic wireless communication effects such as fading and shadowing and therefore cannot address the interplay between sensing, communication, and motionplanning in cooperative networks.

On the communication side, a rich literature has been developed over the years to characterize and model wireless channels, with the cellular industry as one of the key motivations [9-14]. However, the knowledge available on link modeling is spread out over a large body of literature. This makes finding simple yet realistic link models for 


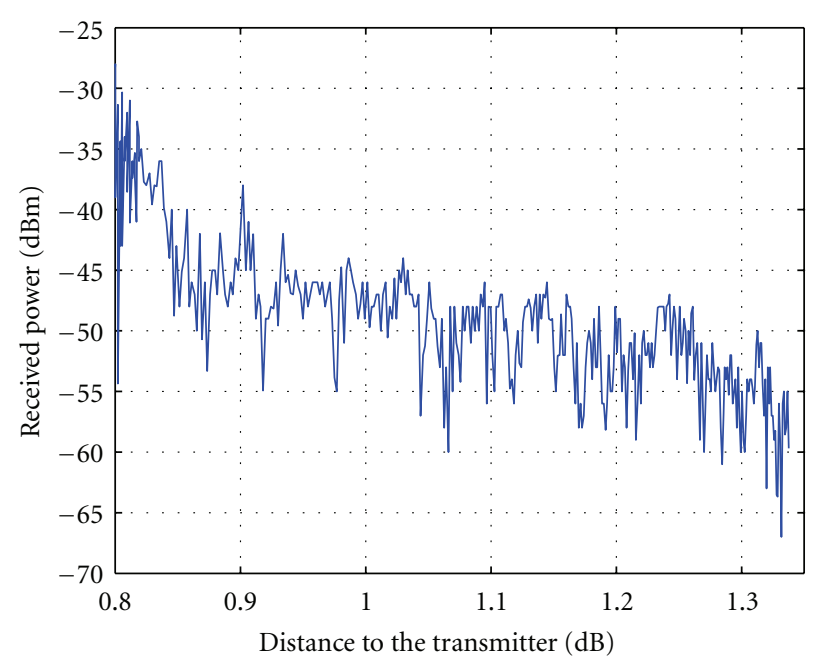

(a)

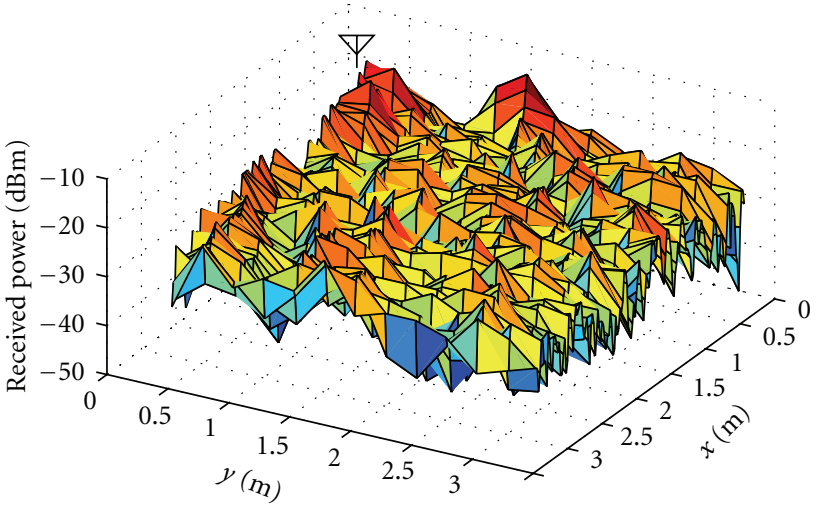

(b)

FIGURE 1: Sample channel measurements in (a) 1D and (b) 2D.

networked robotic applications challenging. Furthermore, the height of the antennas used in robotic applications is typically considerably shorter than those used in most of the traditional wireless communication literature. As such, the applicability of the older models may need further validations. Finally, in robotics, the control of motion as well as location awareness presents new possibilities in terms of improving both the communication quality and channel measurement process, which differentiate these applications from cellular systems or Wireless Local Area Networks (WLANs). For instance, the process of channel measurement can be fully automated. Furthermore, a robot can plan its motion intelligently in order to ensure maintaining connectivity or smart adaptive directional antennas can be utilized in order to reduce the impact of multipath fading.

These are the main motivations for this overview paper. We first summarize a framework for understanding, abstraction, and probabilistic modeling of wireless channels, for networked robotic and control applications, by tapping into the relevant knowledge available in wireless communication literature. We furthermore use a robotic testbed and make several channel measurements in order to assert these mathematical models for the heights and frequencies relevant to robotic applications. We then summarize a few recent examples where this probabilistic characterization was used for the analysis and design of networked robotic systems. Finally, we show how to develop a realistic yet simple channel simulation environment for the verification of cooperative robotic operations in the presence of realistic communication links.

We start in Section 2 by describing our experimental setup for automating the channel measurement process, using our robots. We then summarize a probabilistic characterization of wireless channels and their underlying dynamics/spatial correlation in Section 3 and verify it with experimental measurements. Our mathematical framework is based on well-known references in wireless communication literature such as $[8,14-17]$. We furthermore show the potentials of directional adaptive antennas for improving the link quality in robotic networks. In Sections 4 and 5, we show how the aforementioned probabilistic framework has been recently used for the mathematical analysis and optimization of networked robotic systems, in the presence of realistic links. In Section 6, we show how this framework can be used to develop a simple yet realistic channel simulation environment for networked robotic applications. We conclude in Section 7.

\section{An Experimental Robotic Platform for Channel Measurement Collection}

The analyses of this paper are all accompanied by experimental validations. As such, in this section we briefly describe our experimental testbed. This can help the readers understand the conditions under which our measurements are collected so that they can reproduce the results.

Traditionally, there has been considerable interest in measurement and characterization of the received communication signal strength in the context of cellular systems [18-22]. Automating the measurement process, however, has been difficult in the past due to the lack of an automated mobile system. For outdoor measurements, vehicle-mounted transceivers have been used in some experiments [21, 22]. Collecting indoor measurements, however, is more challenging. For instance, in [18], the authors use a cart to move the receiver and transmitter units, resulting in a positioning accuracy of about $10 \mathrm{~cm}$, which may not suffice depending on the required analysis. Using rails with motorized positioners is another common approach for moving the transmitter/receiver [23]. The advent of robotic networks facilitates the design of an automated measurement system considerably and allows for collecting measurements with flexibility, reconfigurability, and a high spatial resolution. As such, we have developed a robotic 


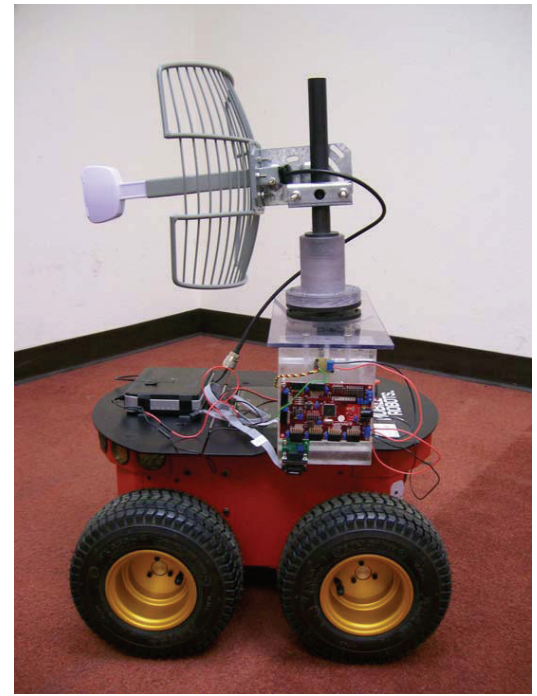

(a)

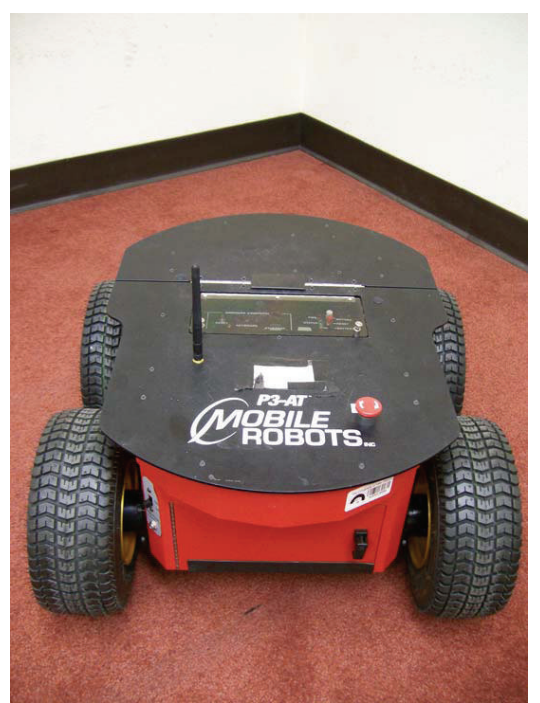

(b)

Figure 2: (a) Pioneer 3-AT robot equipped with a servomechanism and a directional antenna. (b) Pioneer 3-AT robot equipped with an omnidirectional antenna.

testbed to automate our channel measurement process. The testbed consists of two Pioneer 3-AT (P3-AT) mobile robots from MobileRobots Inc. [24], each equipped with an onboard PC, an IEEE 802.11 g (WLAN) card, and various sensors used for localization and obstacle avoidance. Each robot acts as a mobile transceiver and can record its received signal strength as it moves. The resulting data set is then used for the characterization of wireless channels for mobile robotic networks.

Next, we explain the hardware and software components of our testbed in more details including our software-based controller and navigation infrastructure.

2.1. Hardware Architecture. Our setup consists of two P3AT mobile robots [24]. Pioneer 3-AT is a high-performance robotic platform from MobileRobots, which is a popular and reliable team performer for indoor, outdoor, and roughterrain projects. We equipped each robot with a removable electromechanical fixture to possibly hold a directional antenna. Figure 2(b) shows one of our robots in its original form, while Figure 2(a) shows the robot with a directional antenna mounted on it. A block diagram of the hardware architecture of one of the robots is shown in Figure 3. The remote $\mathrm{PC}$ is a supervising unit, in charge of planning the motion of the robots and collecting the signal strength data from the robots. Each P3-AT base comes with an onboard PC104 and a Renesas SH7144-based microcontroller platform to control the motors, actuators, and sensors. MobileRobots provides a $\mathrm{C} / \mathrm{C}++$ application programming interface (API) library called ARIA [24] to program and control the robot via its onboard microcontroller platform. We also developed a servomechanism to intelligently rotate the directional antenna of the robot. The servo mechanism is controlled by the onboard PC of the robot through a microcontroller-based external hardware. We make use of Hitec HS-7955TG high-performance coreless digital servo motors with $180^{\circ}$ rotation in our servomechanism. As for the directional antennas, we use a GD24-15 2.4 GHz parabolic grid antenna from Laird Technologies [25]. This model has a $15 \mathrm{dBi}$ gain with $21^{\circ}$ horizontal and $17^{\circ}$ vertical beamwidth and is suitable for IEEE $802.11 \mathrm{~b} / \mathrm{g}$ applications (Figure 2(a)).

2.1.1. Robot Localization. Accurate localization of the robots is crucial to proper channel measurement and analysis. For instance, characterizing the spatial correlation of different channel dynamics requires accurate position information. In our testbed, each robot uses both the onboard gyroscope and the wheel encoders for localization. Since the localization error is additive in time, the calibration unit resets the gyroscope and the wheel encoders periodically, after an adjustable number of steps. Currently, our localization error is less than $2.5 \mathrm{~cm}$ for every $1 \mathrm{~m}$ of a straight line movement. If additional accuracy is needed over longer distances, more advanced localization strategies, from the robotic literature, can also be utilized. Alternatively, a long route can be divided into shorter subroutes and the robot can be manually repositioned at the beginning of each sub-route to provide a better overall accuracy.

2.1.2. Software Architecture. A high-level schematic of the software architecture is shown in Figure 4. The softwarebased control infrastructure consists of two application layers running on different machines: the robot-side application runs on the onboard PC of the robot whereas the clientside application runs on the remote PC. the robot-side application is developed as a TCP/IP server and is in charge of reading the sensory data, sending it to the client-side application, receiving the high-level control of motion/antenna angle commands from the client-side application and executing the commands. The client-side application, which runs as a TCP/IP client for robot-side application, is in charge of supervising the entire operation, planning the 


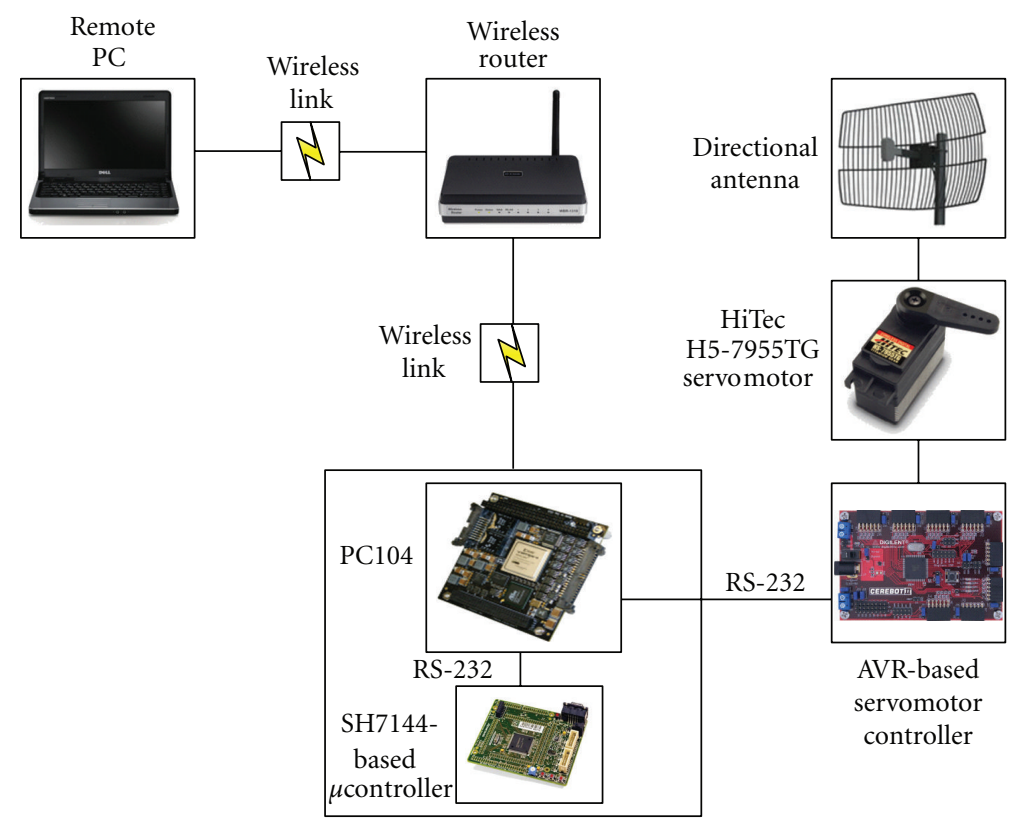

FIGURE 3: A block diagram of the hardware architecture of one of the robots.

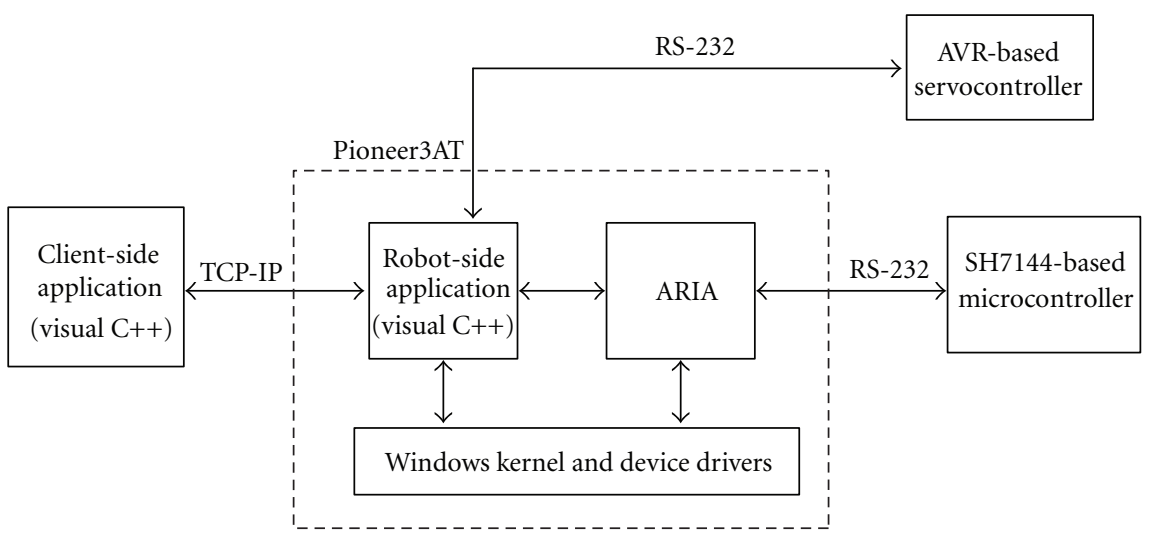

FIgURE 4: The overall software architecture of the robotic platform.

motion, generating the high-level control commands to be sent to the robots, and collecting the signal strength data from the robots for future processing. The microcontroller of the servo mechanism is also programmed to decode the rotation commands and send the corresponding Pulse Width Modulation (PWM) signals to the servo motor that rotates the antenna. The operating system is Microsoft Windows XP, and all the programs are developed in $\mathrm{C}++$ using MS Visual Studio 2008. The user can run both robots simultaneously, calibrate and test the servo mechanism, and run several automatic data gathering scenarios. Among all the possible scenarios, the following two are used extensively for the analysis presented in this paper.

(i) Scenario 1. The transmitter is a wireless $802.11 \mathrm{~g}$ router with an omnidirectional antenna at a height of $1.5 \mathrm{~m}$. The receiver is a robot with an omnidirectional antenna at the height of $27 \mathrm{~cm}$ (see Figure 2(b)). (ii) Scenario 2. Both the transmitter and receiver are robots with different combinations of directional/ omnidirectional TX/RX antennas. The directional antenna is as shown in Figure 2(a).

Unless stated otherwise, the default mode of operation is scenario 1 throughout the paper.

\section{Characterization of the Spatial Variations of a Wireless Channel $[8,16]$}

In this section, our goal is to summarize the existing results on the probabilistic characterization of wireless channels, from the wireless communication literature, and to confirm this characterization with our robots. In general, exact mathematical characterization of a wireless channel is extremely challenging, due to its time-varying and unpredictable nature. Blocking, reflection, scattering, and diffraction are 


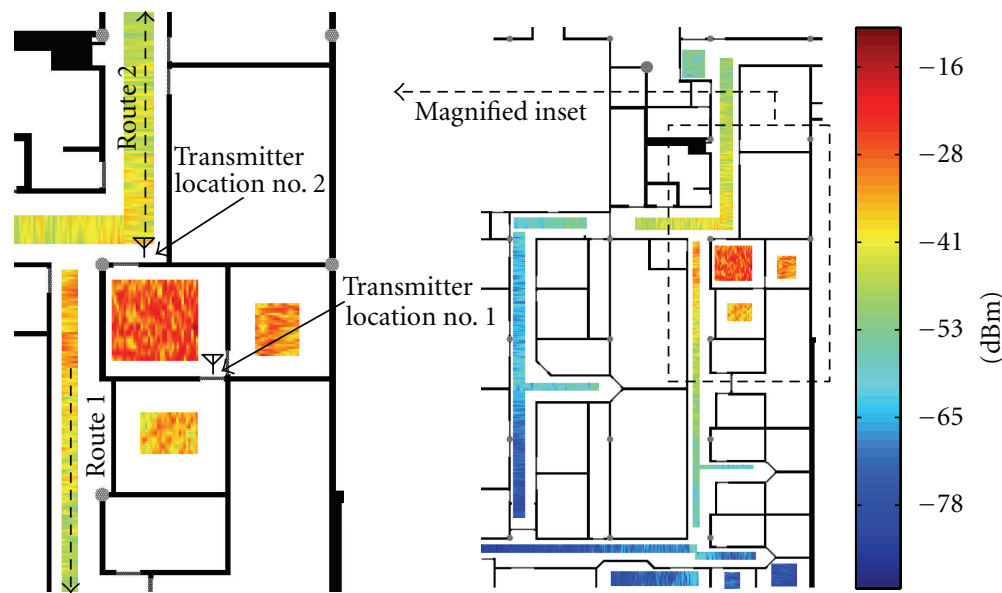

(a)

(b)

FIGURE 5: (b) Blueprint of the basement of the ECE bulding. at UNM where channel measurements are collected-a colormap of the measured received signal power is superimposed on the map for the transmitter at location \#1. (a) A magnified inset of the blueprint.

a few examples of phenomena that a transmitted wave between two robotic agents can experience. One can possibly solve Maxwell's equations with proper boundary conditions that reflect all the physical constraints of the environment. However, such calculation is difficult and requires the knowledge of several geometric and dielectric properties of the environment, which is not easily available. In wireless communication systems, it is therefore common to model the channel probabilistically, with the goal of capturing its underlying dynamics. The utilized probabilistic models are the results of analyzing several empirical data over the years. In general, a communication channel between two robotic platforms can be modeled as a multiscale dynamical system with three major dynamics: small-scale fading (multipath fading), shadowing (shadow fading), and path loss. These three dynamics are key to the realistic characterization of the performance of networked robotic systems. We first show an example of these three dynamics through an experiment with our robotic testbed.

Figure 5 shows the blueprint of the basement of the Electrical and Computer Engineering building at UNM where we made several measurements along more than 70 routes using the experimental setup described in Section 2. (Unless we specifically indicate otherwise, both the transmitter and receiver antennas should be assumed omnidirectional (scenario 1). In such cases, the transmitter is a fixed router at the height of $1.5 \mathrm{~m}$ and the receiver is a moving robot with its antenna at the height of $27 \mathrm{~cm}$.) The figure also shows a colormap of our measured received signal power for the transmitter at location no. 1. In this paper, we use this data for analysis and mathematical characterization. It should, however, be noted that the framework of this paper is also fully applicable for modeling outdoor wireless measurements. We used indoor measurements in this paper since wireless link quality is typically worse inside a building (due to the higher chance of lacking a line-of-sight communication).

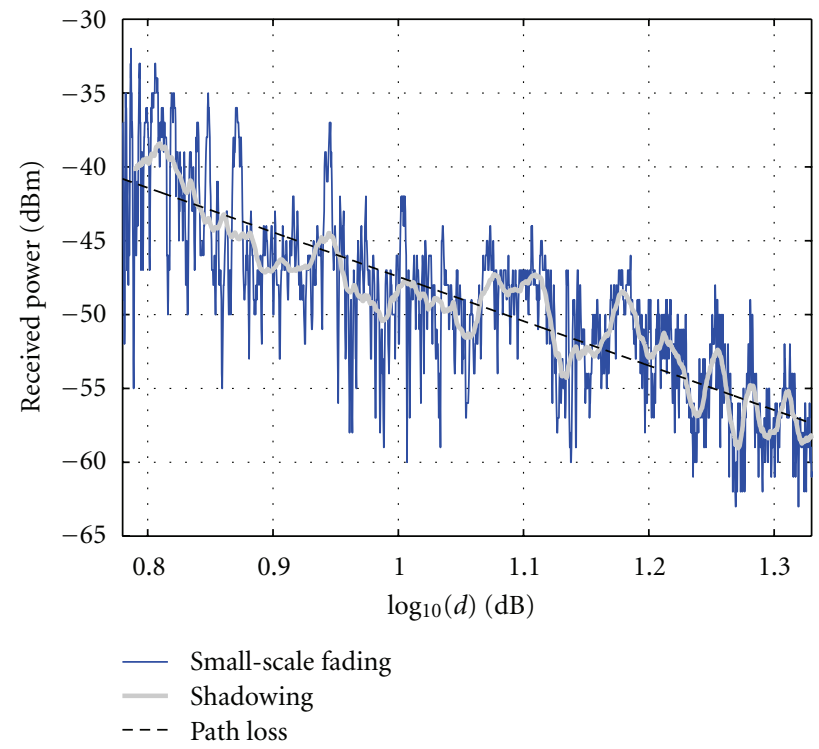

Figure 6: Underlying dynamics of the received signal power across route 1 of Figure 5 and for the transmitter at location \#1. The blue curve is the measured received power which exhibits smallscale fading. By averaging locally over small-scale variations, the underlying shadowing variations can be seen (gray). The average of the shadowing variations then follows the distance-dependent path loss curve (dashed line).

As an example, Figure 6 shows the received signal power across route 1, as marked in Figure 5, for the transmitter at location $\# 1$ and as a function of the distance to the transmitter. The three main dynamics of the received signal power are marked on the figure. As can be seen, the received power can have rapid spatial variations that are referred to as small-scale fading. By spatially averaging the received signal locally and over distances where channel can still be considered stationary, a slower dynamic emerges, which is 
called shadowing. Finally, by averaging over the variations of shadowing, a distance-dependent trend is seen, which is referred to as path loss. In this section, we provide an understanding and modeling of these underlying dynamics.

3.1. Small-Scale Fading (Multipath Fading). When a wireless transmission occurs, replicas of the transmitted signal will arrive at the receiver due to phenomena such as reflection and scattering. This results in the following baseband equivalent channel at time instant $t$ :

$$
h(t)=\sum_{n=1}^{N(t)} \alpha_{n}(t) e^{j \varsigma_{n}(t)-j 2 \pi f_{c} \tau_{n}(t)}
$$

where $N(t)$ represents the total number of paths that arrive at the receiving robot at time $t, f_{c}$ is the carrier frequency, and $\alpha_{n}, \tau_{n}$, and $\varsigma_{n}$ are the attenuation, delay, and Doppler phase shift of the $n$th path respectively. As can be seen from (1), different paths can be added constructively or destructively depending on the phase terms of individual paths. As a result, with a small movement, the phase terms can change drastically, resulting in the rapid variations of the channel. Such rapid variations are referred to as small-scale fading (multipath fading) and can be seen in Figure 6. The higher the number of reflectors and scatterers in the environment is, the more severe the small-scale variations could be. Next, we characterize the distribution of $|h(t)|$ (which easily translates to a distribution for the received Signal-to-Noise Ratio (SNR) since it is proportional to $|h(t)|^{2}$ ).

In the wireless communications literature, several efforts have been made in order to mathematically characterize the behavior of small-scale fading. As can be seen from Figure 6, the small-scale fading curve is nonstationary over large distances as its average is changing. Therefore, it is common to characterize the behavior of it over small-enough distances where channel can be considered stationary. Then, the behavior of the average of the small-scale variations is characterized in order to address channel dynamics over larger distances, as we shall see in the next part. Over small-enough distances where channel (or equivalently the received signal power) can be considered stationary, it can be mathematically shown that Rayleigh distribution is a good match for the distribution of $|h(t)|$ if there is no Line-OfSight (LOS) path while Rician provides a better match if an LOS exists. These distributions also match several empirical data. A more general distribution that was shown to match empirical data is Nakagami distribution $[15,16,26]$, which has the following pdf for $z(t)=|h(t)|$ :

$$
p(z)=\frac{2 m^{m} z^{2 m-1}}{\Gamma(m) \bar{P}_{z}^{m}} \exp \left[\frac{-m z^{2}}{\bar{P}_{z}}\right], \quad \text { for } z \geq 0
$$

where $m \geq 0.5$ is the fading parameter, $\bar{P}_{z}=\mathbb{E}\left[|h(t)|^{2}\right]$ represent the average power of the channel (averaged over smallscale fading), and $\Gamma(\cdot)$ is the Gamma function. If $m=1$, this distribution becomes Rayleigh: $p^{\text {ray }}(z)=2 z / \bar{P}_{z} \exp \left[-z^{2} / \bar{P}_{z}\right]$, for $z \geq 0$, whereas for $m=\left(m^{\prime}+1\right)^{2} /\left(2 m^{\prime}+1\right)$, it is approximately reduced to a Rician distribution with parameter $m^{\prime}$ :

$$
\begin{aligned}
p^{\mathrm{ric}}(z)= & \frac{2 z\left(m^{\prime}+1\right)}{\bar{P}_{z}} \exp \left[-m^{\prime}-\frac{\left(m^{\prime}+1\right) z^{2}}{\bar{P}_{z}}\right] \\
& \times I_{0}\left(2 z \sqrt{\frac{m^{\prime}\left(m^{\prime}+1\right)}{\bar{P}_{z}}}\right),
\end{aligned}
$$

for $z \geq 0$. Similarly, distributions of the power of the channel $\left(|h|^{2}\right)$, the received power, and SNR can be derived by a change of variables. Such distributions can be very helpful in generating realistic communication links for the purpose of mathematical analysis, optimization as well as simulation in robotic networks.

We verified the Nakagami distribution using several measurements in our building. While Rayleigh and Rician distributions are more heavily assumed for the purpose of analysis involving wireless channels, we found that a general Nakagami distribution is a better match for most of our gathered data. As an example, consider the measurement of Figure 6, which is across route 1 of Figure 5 and for the transmitter at location \#1. Figure 7 shows the probability density function (pdf) and cumulative distribution function (cdf) of three different sections of the small-scale variations across this route. These parts are chosen such that the data can be considered stationary within each section (since small-scale analysis is only relevant to the small-enough and thus stationary parts). It can be seen that the distribution of the gathered data matches power distribution for Nakagami fading with parameters $m=1.20$ and $m=1.30$ well. Note that since the distribution of the power of the received signal, which is proportional to $|h(t)|^{2}$, is plotted, the figure does not show a Nakagami distribution directly. It shows the power distribution of Nakagami fading, that is, the distribution of a nonnegative variable whose square root has a Nakagami distribution.

While Nakagami distribution shows a good match for the distribution of small-scale fading, mathematical analysis of the performance of a robotic network under such a distribution is generally challenging. Alternatively, a simpler but suboptimum match is lognormal. In [27], the authors showed that a Gaussian distribution can possibly provide an acceptable match for the distribution of the small-scale variations in $\mathrm{dB}$ (albeit with some loss of performance as compared to Nakagami). Figure 8 compares the match of both Nakagami and lognormal to the distribution of smallscale fading for two different stationary sections of the data of Figure 6. As can be seen, Nakagami provides a considerably better match while lognormal can be acceptable depending on the required accuracy.

3.2. Shadow Fading (Shadowing). As discussed in the previous part, the received wireless signal is nonstationary over large distances. While small-scale fading characterizes the behavior of the channel over a small distance, it does not suffice for characterizing the channel over larger distances. Small-scale variations are the result of a number of paths arriving at the receiver at approximately the same time 


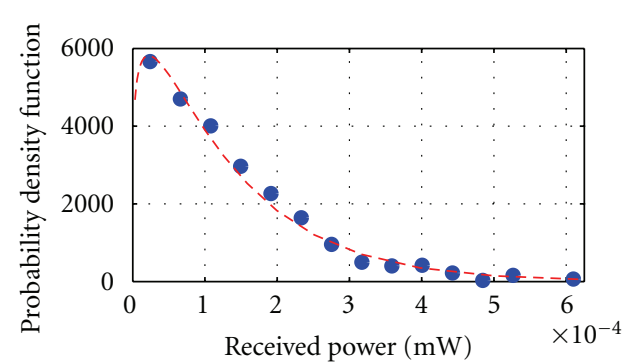

- Experimental data

- - Power distribution of Nakagami fading with $m=1.2$

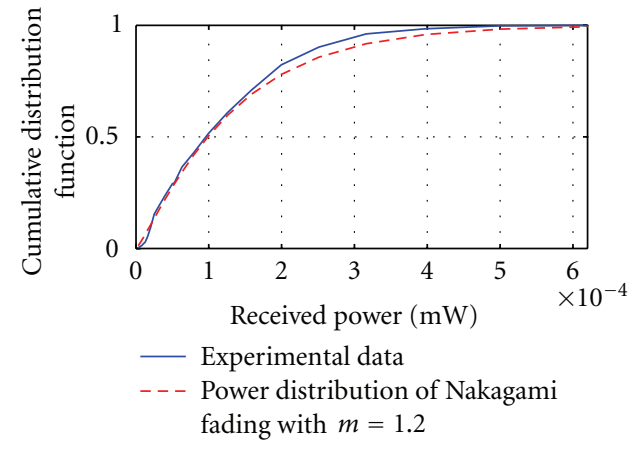

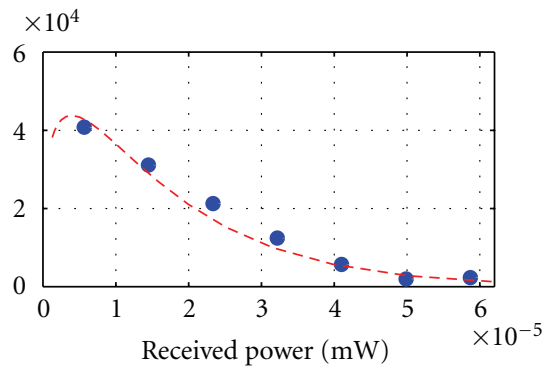

- Experimental data

- - Power distribution of Nakagami fading with $m=1.3$

(a)
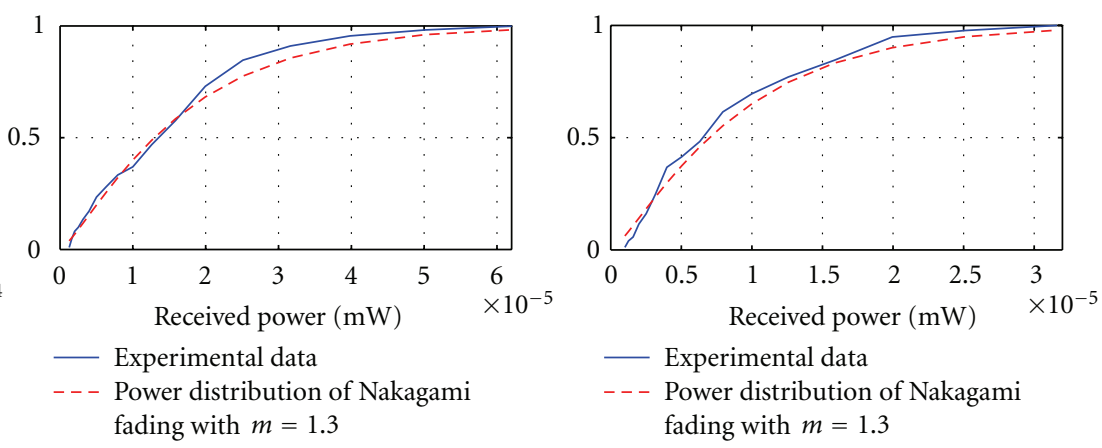

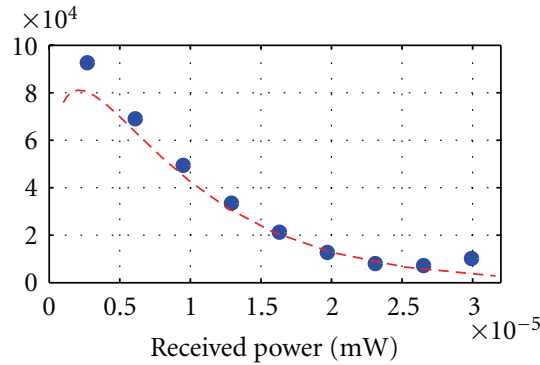

- Experimental data

- - Power distribution of Nakagami fading with $m=1.3$

(b)

FIGURE 7: The distribution of small-scale fading using three different parts of our gathered measurements. Nakagami distribution shows a very good match-(a) pdf and (b) cdf.

but being added constructively or destructively, depending on their phase terms, which results in rapid variations. As Figure 9 shows, once we average over small-scale variations, another dynamic can be observed which changes at a slower rate. Let $\bar{P}_{z}=\mathbb{E}\left[|h(t)|^{2}\right]$ represent the average power of the channel (averaged over small-scale fading), as defined for (2). This signal varies over larger distances and is referred to as shadow fading or shadowing. Shadowing is the result of the transmitted signal being possibly blocked by a number of obstacles before reaching the receiver. Empirical data has shown $\bar{P}_{z}$ to have a lognormal distribution (mathematical justification also exists by using Central Limit Theorem [16]). Let $\bar{P}_{z, \mathrm{~dB}}=10 \log _{10}\left(\bar{P}_{z}\right)$. We have the following for the distribution of $\bar{P}_{z, \mathrm{~dB}}[15,16,28,29]$ :

$$
p\left(\bar{P}_{z, \mathrm{~dB}}\right)=\frac{1}{\sqrt{2 \pi} \sigma_{\mathrm{dB}}} e^{-\left(\bar{P}_{z, \mathrm{~dB}}-\mu_{\mathrm{dB}}\right)^{2} / 2 \sigma_{\mathrm{dB}}^{2}},
$$

where $\mu_{\mathrm{dB}}=K_{\mathrm{dB}}-10 \gamma \log _{10}(d)$ and $\sigma_{\mathrm{dB}}$ is the standard deviation of $\bar{P}_{z, \mathrm{~dB}}$. Consider the distance-dependent path loss, $\mu=K / d^{\gamma}$, where $d$ represents the distance between the transmitting and receiving robots, $\gamma$ denotes the power falloff rate, and $K>0$ is a constant. Then, it can be seen from (4) that $\mu_{\mathrm{dB}}=10 \log _{10}(\mu)=K_{\mathrm{dB}}-10 \gamma \log _{10}(d)$ represents the average of shadowing variations. Note that average SNR will also have a lognormal distribution.
Figure 10 shows the pdf and cdf of shadow fading for all the collected data in the basement of ECE building, as shown in Figure 5, and for the transmitter at location \#1. In order to access the shadowing variations, the gathered data of each route is averaged locally over small-scale fading, as illustrated in Figure 9. It should be noted that the resulting shadowing variation is non-stationary as its average changes with distance. The distance-dependent path loss component for each route can be easily estimated by finding the best linear fit that relates the log of the received power of the collected data to the log of the distance traveled (see Figure 6 for an example). We then remove the distance-dependent average from shadowing variations before characterizing the distribution of the collected data. As a result, the distribution of the resulting gathered data should match a zero-mean lognormal distribution. It can be seen from Figure 10 that the distribution of the log of the shadowing variations (after removing the distance-dependent average) matches a zeromean normal distribution very well. The three columns correspond to averaging window sizes of $0.4 \lambda, 1 \lambda$, and $10 \lambda$ from left to right, where $\lambda$ is the wavelength of operation. The standard deviations for these matches are $\sigma_{\mathrm{dB}}=2.7$, $\sigma_{\mathrm{dB}}=2.3$, and $\sigma_{\mathrm{dB}}=1.4$, respectively. As can be seen, as the averaging window size increases, the standard deviation of the best fit becomes smaller. This is as expected since, by averaging over larger distances, the resulting signal becomes 


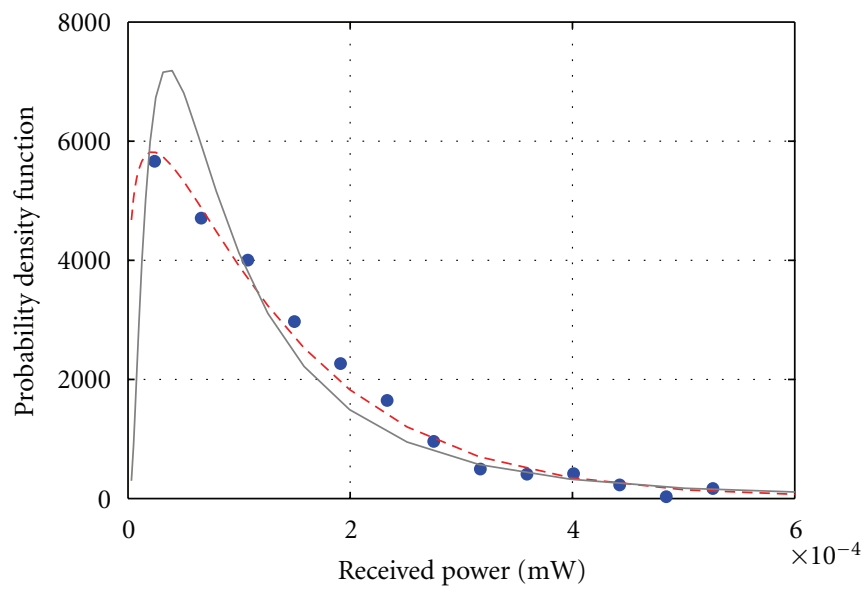

- Experimental data

- - - Power distribution of Nakagami fading with $m=1.2$

Lognormal fit

(a)

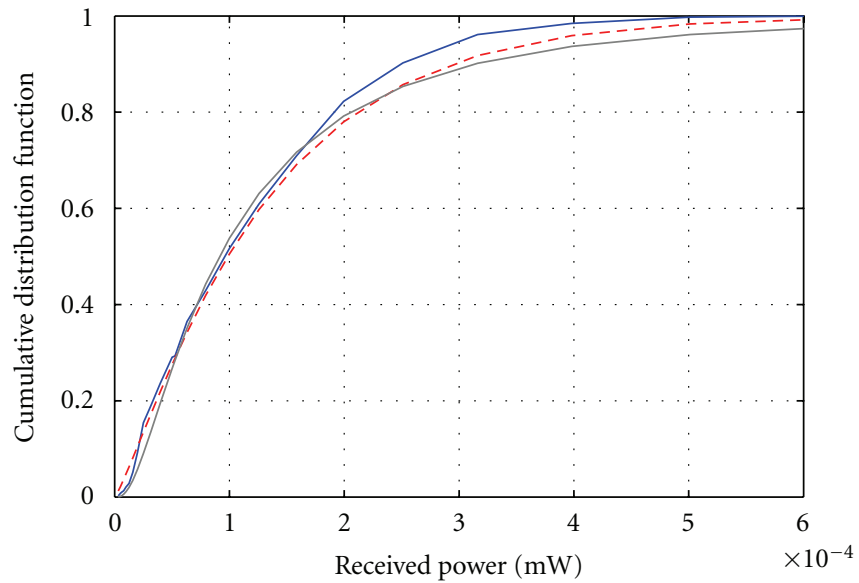

- Experimental data
- - Power distribution of Nakagami fading with $m=1.2$
_ Lognormal fit

(b)

FIGURE 8: Comparison of Nakagami and lognormal for the distribution of small-scale fading-(a) pdf and (b) cdf.

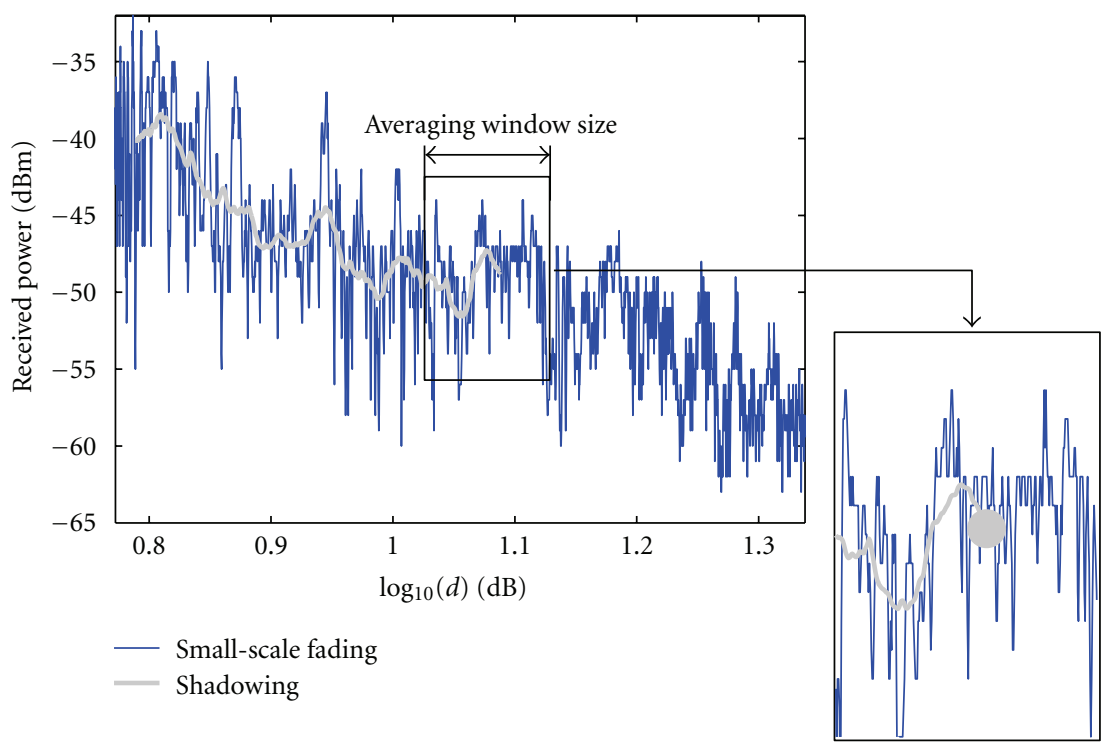

FIGURE 9: Illustration of moving average over small-scale variations in order to obtain shadowing dynamics. An appropriate window length is chosen such that the small-scale variations can be considered stationary over that length. Then, the value of shadowing at the center of this window corresponds to the average of all the data points within the window. Alternatively, the window size can be adaptive.

closer to the underlying overall average (distance-dependent path loss). For this specific data, the best fit corresponds to the averaging window size of $0.4 \lambda$, with a Normalized Mean Square Error of $2.89 \times 10^{-4}$.

3.3. Distance-Dependent Path Loss. It can be seen from (4) that the distance-dependent path loss, characterized as $K_{\mathrm{dB}}-$ $10 \gamma \log _{10}(d)$, is the average of the shadowing variations. This completes the relationship between the three underlying dynamics: small-scale fading, shadow fading, and path loss. As mentioned earlier, the distance-dependent path loss component can be found by finding the best linear fit that relates the $\log$ of the received signal power to the $\log$ of the distance traveled. For instance, for the data of Figure 6, path loss component can be characterized as $-17.35-$ $30 \log _{10}(d)$. It should be noted that the parameters of path loss curve, such as exponent $\gamma$, vary from route to route. They can even vary within a route if the route is considerably long.

In current networked robotics literature, it is common to use fixed-radius disc models to model wireless channels. It is noteworthy that this over-simplified model only considers 


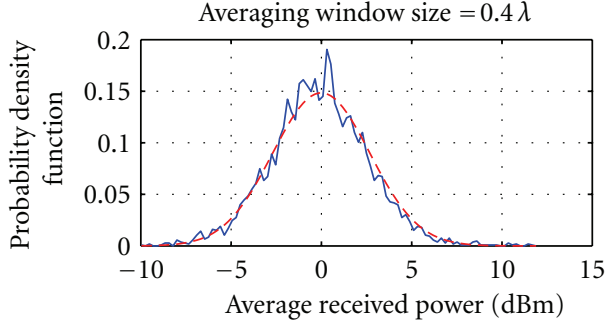

(a)

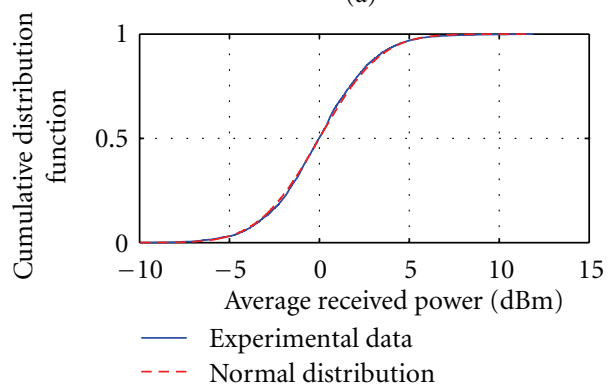

(d)

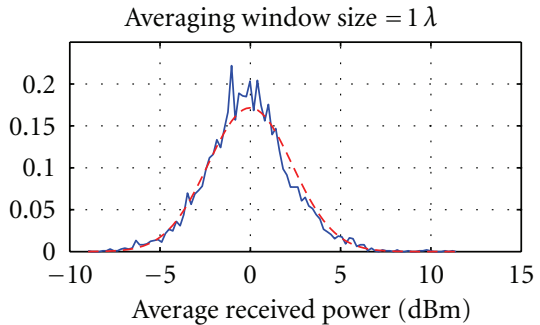

(b)

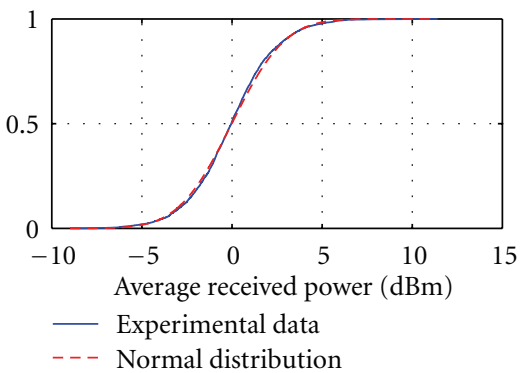

(e)

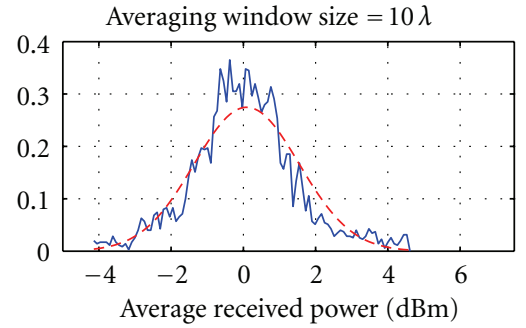

(c)

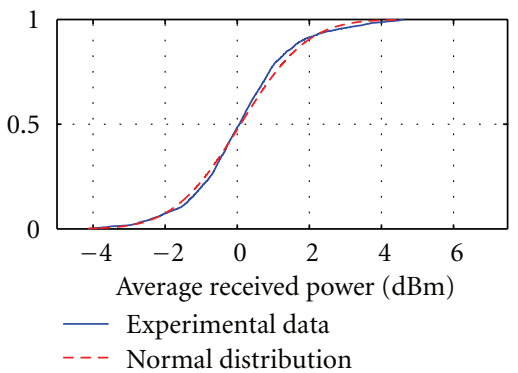

(f)

FIGURE 10: $(\mathrm{a}-\mathrm{c})$ pdf and $(\mathrm{d}-\mathrm{f}) \mathrm{cdf}$ of the log of shadow fading (after removing the distance-dependent path loss) and the normal distribution match for all the data gathered in the basement of ECE bulding. The three columns show the impact of the averaging window size on the match: (a, d) window size of $0.4 \lambda$, (b, e) window size of $1.0 \lambda$, and (c, f) window size of $10.0 \lambda$, with $\lambda=0.125 \mathrm{~m}$ denoting the wavelength of the transmitted signal.

path loss. It furthermore assumes the same path loss parameters everywhere in the environment. Therefore, it is only a very crude representation after considerable averaging is done.

3.4. Channel Spatial Correlation. Thus far we characterized the distribution of a wireless channel at a single position (or equivalently at a time instant). Another important parameter that characterizes a wireless channel is its spatial correlation, that is, how fast the small-scale and shadow fading components are changing spatially. Channel spatial correlation plays a critical role in the cooperative operation of autonomous agents. For instance, it impacts how well we can predict channel spatial variations [30-32] and embed the corresponding communication objectives in a motionplanning function [30,32].

Spatial correlation of small-scale fading depends on the speed of the robots, frequency of operation, and antenna beamwidth/gain, among several other factors. The least correlation is typically observed when there exists a rich scatterer/reflector environment that results in a uniform angle of arrival of the paths. In such cases, the power spectrum of small-scale fading will have a form that is referred to as Jakes spectrum [8] and channel decorrelates on the order of $0.4 \lambda$, with $\lambda$ representing the wavelength ( $5 \mathrm{~cm}$ for $2.4 \mathrm{GHz}$ WLAN transmission). If this is not the case, the spatial correlation function of small-scale fading can be mathematically derived for more general cases [8]. However, a general model that can fit several scenarios does not exist. For most scenarios, small-scale fading decorrelates considerably fast, as compared to the other dynamics.
For shadow fading, there is less mathematical characterization of spatial correlation. Gudmundson [33] characterizes an exponentially decaying spatial covariance function for the log of the shadow-fading variations, based on outdoor empirical data, which is widely used:

$$
\begin{aligned}
A_{\mathrm{cov}, \bar{P}_{z, \mathrm{~dB}}}\left(\left\|q_{1}-q_{2}\right\|\right) & =\mathbb{E}\left[\left(\bar{P}_{z, \mathrm{~dB}, 1}-\mu_{\mathrm{dB}, 1}\right)\left(\bar{P}_{z, \mathrm{~dB}, 2}-\mu_{\mathrm{dB}, 2}\right)\right] \\
& =\sigma_{\mathrm{dB}}^{2} e^{-\left\|q_{1}-q_{2}\right\| / X_{c}}, \quad q_{1}, q_{2} \in \mathbb{R}^{2},
\end{aligned}
$$

where $\bar{P}_{z, \mathrm{~dB}, 1}$ and $\bar{P}_{z, \mathrm{~dB}, 2}$ are the average power of the channel (averaged over small-scale fading) at positions $q_{1}$ and $q_{2}$, respectively, $\mu_{\mathrm{dB}, 1}$ and $\mu_{\mathrm{dB}, 2}$ are the corresponding path loss components, $\sigma_{\mathrm{dB}}^{2}$ is the variance of the $\log$ of shadowing as defined in (4), and $X_{c}$ is the decorrelation distance, which is defined as the distance at which the autocovariance reaches $1 / e$ of its maximum value. It has been shown that the decorrelation distance is on the order of the size of the blocking objects or clusters of objects [16].

We used our channel measurements and found the exponential to be a good match for the correlation of shadowing. Figure 11 shows the normalized autocovariance function for the data gathered in route 2 of Figure 5 with the transmitter at location \#1. It can be seen that the real autocovariance function matches the exponential model considerably well although this is an indoor measurement. We see similar matches across other routes of Figure 5.

3.5. Impact of Antenna Angle. As seen in the previous sections, small-scale fading can result in the severe fluctuations of the received signal power, which can degrade the 


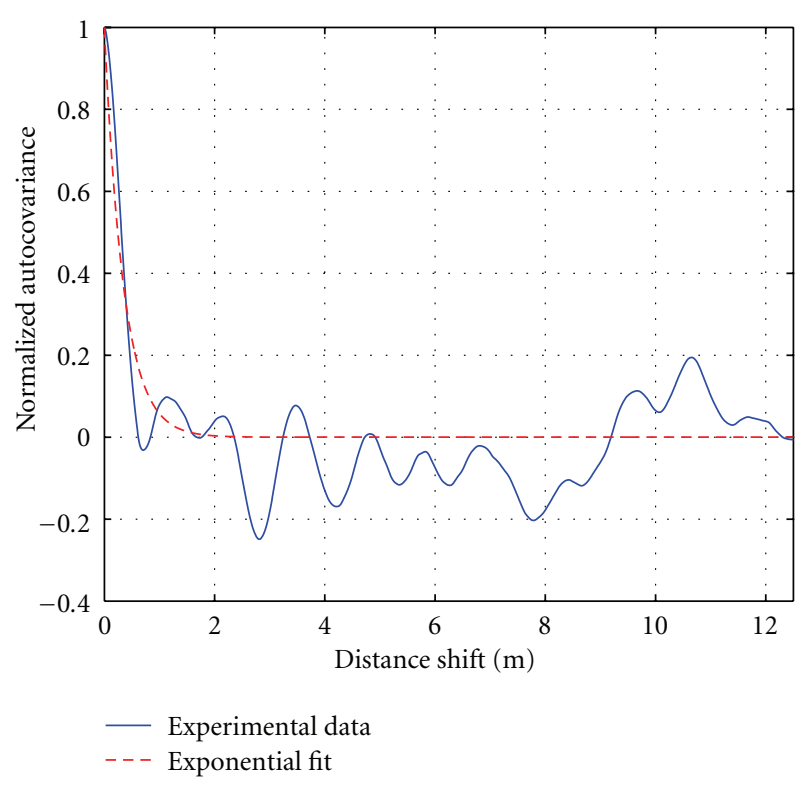

FIGURE 11: Exponential match for the normalized autocovariance of the log of shadowing variations. It can be seen that exponential provides a good match.

performance of a robotic network considerably. The main contributor to such fluctuations is the fact that different multipaths can be added constructively or destructively depending on their traveled routes. One possible way to mitigate the impact of multipath fading is to use adaptive directional antennas with a small beamwidth (angle). A smaller beamwidth can limit the number of multipaths that reach the receiver, which will reduce the chance of the paths being added out of phase. This approach, however, would require alignment and adaption of the transmitting and receiving antennas in order to make sure that they face each other when communicating. As such, it does not work for nonrobotic communication systems, such as cellular systems or Wireless Local Area Networks (WLANs), where control of angle is simply not possible. In a robotic network, however, the angle can be adapted. Each robot typically knows the position of another robot in the network, which can be used for online adaption and alignment of directional antennas.

We have also equipped our robots with adaptive directional antennas in order to see their impact on multipath fading. Figure 12(a), for instance, shows an operation using an adaptive and an omnidirectional antenna whereas, in Figure 12(b), both robots are using adaptive antennas. Figure 13 shows the impact of small antenna beamwidth on small-scale fading. The figure shows the received signal power across route 2, marked on Figure 5, and for the transmitting robot at location \#2. In the omni-to-omni case, both the transmitter and receiver are omnidirectional. In the omni-to-dir case, the transmitter is omnidirectional while the receiver is directional. Finally, for the dir-to-dir case, both the transmitter and receiver are directional. Our directional antenna has a horizontal and vertical beamwidth of $21^{\circ}$ and $17^{\circ}$, respectively.
It can be seen that the dir-to-dir case results in the smallest amount of variations. To measure this, the standard deviations of the received signal power from the distancedependent path loss are calculated to be 4.53, 2.44, and 1.89 for the omni-to-omni, omni-to-dir, and dir-to-dir, cases, respectively. Furthermore, it can be seen that the overall signal power increases as we use directional antennas. We saw similar behaviors across other routes in our building. This shows the potential of directional adaptive antennas for networked robotic applications.

\section{Probabilistic Analysis of Networked Robotic Systems in the Presence of Realistic Wireless Channels}

In the robotics literature, oftentimes disk models are used to characterize wireless channels when theoretically analyzing the behavior of a networked robotic and control system. Performing theoretical analysis with such a model, however, can lead to conclusions that are far from the true behavior of these systems. The probabilistic characterization of the previous section, on the other hand, can be used for more accurate mathematical analysis of networked robotic systems. For instance, wireless links can be modeled as random nonstationary processes (small-scale fading) with a Rayleigh, Rician, or Nakagami distribution. Then the average of the power of this process will have a lognormal distribution with a mean that follows the distance-dependent path loss (in $\mathrm{dB})$. As such, the overall behavior of a networked robotic systems, in presence of realistic wireless channels, can be characterized probabilistically. In order to see an example of this, consider a cooperative target tracking operation, where a team of $N_{r}$ robots track a moving target as shown in Figure $14[31,32,34]$. The nodes constantly sense the position of the target and send their sensory information to a fixed base station over wireless links. The overall goal is for the base station to have the best estimate of the position of the target in real time.

Let $\xi(t) \in \mathbb{R}^{2}$ denote the position of the target at time instant $t$. Consider the following linear sensing model for the $j$ th robot, for $j=1, \ldots, N_{r}: \eta_{j}(t)=\xi(t)+v_{j}(t)$, where $\eta_{j}(t) \in \mathbb{R}^{2}$ is the observation of the $j$ th robot and $v_{j}(t) \in \mathbb{R}^{2}$ is a zero-mean Gaussian noise with $\Pi_{j}(t)=\mathbb{E}\left[v_{j}(t) v_{j}^{T}(t)\right]$ representing its covariance matrix. Each node constantly sends its observations to the base station. Depending on the quality of the link from node $j$ to the base station at time $t$, the transmitted observation may be received by the base station or may be dropped due to poor link quality. In other words, if the received power (or equivalently the received SNR) on a given link, at a given time, is below a given threshold $\left(P_{\mathrm{TH}}\right)$, the transmitted information on that link will not be received by the base station. Let $P_{r, j}(t)$ denote the received power at the base station in communication from the $j$ th node at time $t$. Then, the received observation from the $j$ th node is kept if $P_{r, j}(t)>P_{\mathrm{TH}}$ and is dropped otherwise.

At each time step, the base station fuses all the received observations using a Best Linear Unbiased Estimator (BLUE). Let $\hat{\xi}_{b}(t)$ represent the estimate of the base station of 


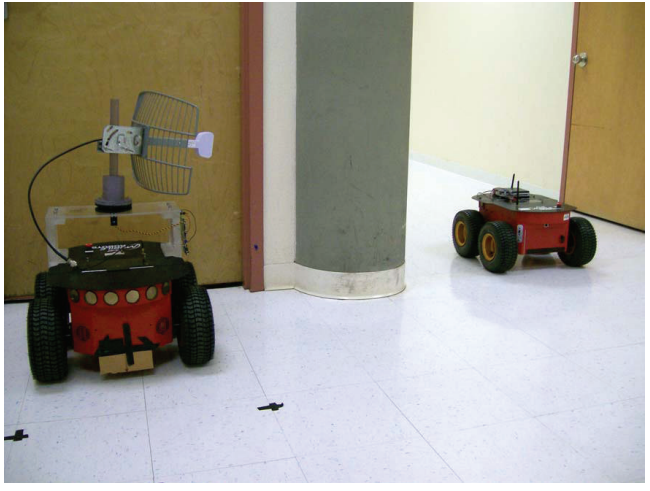

(a)

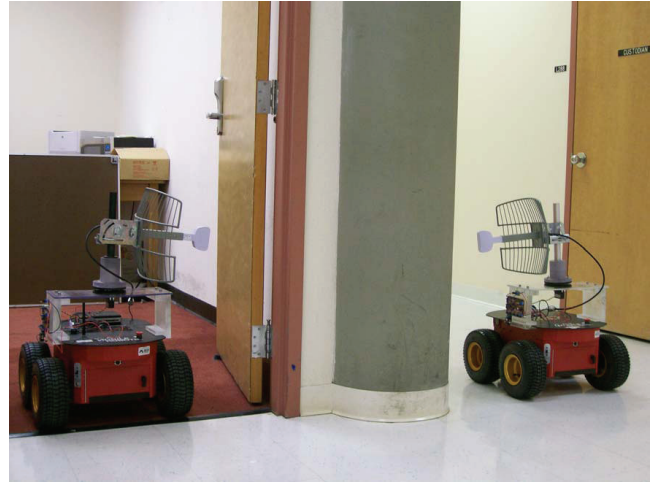

(b)

FIgURE 12: (a) Pioneer robots gathering data at the basement of the ECE building at UNM with the transmitter using an omnidirectional antenna and the receiver using a directional one. (b) Pioneer robots using directional antennas for both transmission and reception.

the position of the target at time $t$, after fusing all the received information, with $\Pi_{b}(t)$ denoting the corresponding error covariance. We have

$$
\begin{aligned}
\hat{\xi}_{b}(t) & =\left[\sum_{j=1}^{N_{r}} \rho_{j}(t) \Pi_{j}^{-1}(t)\right]^{-1}\left[\sum_{j=1}^{N_{r}} \rho_{j}(t) \Pi_{j}^{-1}(t) \eta_{j}(t)\right], \\
\Pi_{b}(t) & =\mathbb{E}\left[\left(\xi(t)-\hat{\xi}_{b}(t)\right)\left(\xi(t)-\hat{\xi}_{b}(t)\right)^{T}\right] \\
& =[\sum_{j=1}^{N_{r}} \rho_{j}(t) \underbrace{\Pi_{j}^{-1}(t)}_{l_{j}(t)}]^{-1},
\end{aligned}
$$

where $\rho_{j}(t)$ is defined as follows:

$$
\rho_{j}(t)= \begin{cases}1 & P_{r, j}(t)>P_{\mathrm{TH}}, \\ 0 & \text { else, }\end{cases}
$$

and $\ell_{j}(t) \triangleq \Pi_{j}^{-1}(t)$ denotes the Fisher information collected by the $j$ th node from the target at time instant $t$ [35]. Depending on the existence of the corresponding link to the base station (i.e., the value of $\rho_{j}(t)$ ), this information may or may not be received by the base station. Let $l_{b}(t) \triangleq \Pi_{b}^{-1}(t)$ denote the overall instantaneous Fisher information received at the base station at time instant $t$. We have

$$
\ell_{b}(t) \triangleq \Pi_{b}^{-1}(t)=\sum_{j=1}^{N_{r}} \rho_{j}(t) \boldsymbol{l}_{j}(t)
$$

Let $\ell_{b, \text { total,traj }} \triangleq \sum_{t=0}^{T_{\max }-1} \ell_{b}(t)$ denote the overall accumulated Fisher information at the base station in a given total number of time steps, $T_{\max }$, and for a given set of trajectories of the robots. We have

$$
\ell_{b, \text { total,traj }}=\sum_{t=0}^{T_{\max }-1} \sum_{j=1}^{N_{r}} \rho_{j}(t) \ell_{j}(t) .
$$

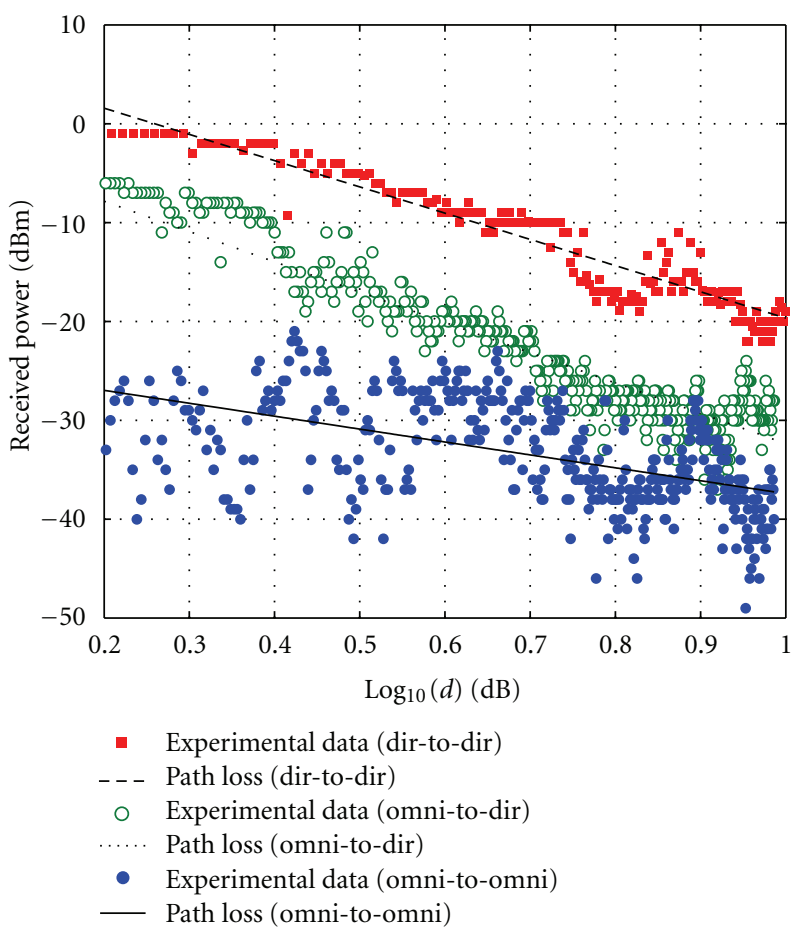

FIGURE 13: Impact of antenna angle in reducing small-scale fading. It can be seen that using an adaptive antenna with a small beamwidth can reduce the amount of multipath fading considerably and also increase the overall received signal power.

Equation (9) is a function of a given set of trajectories of the robots. The trajectories of the robots are dictated by the trajectory of the target. Therefore, we next calculate the average accumulated Fisher information of the base station, averaged over random target and hence robot trajectories. We have

$$
\begin{aligned}
l_{b, \text { total ave }} & \triangleq \mathbb{E}\left[\ell_{b, \text { total,traj }}\right]=\sum_{t=0}^{T_{\max }-1} \sum_{j=1}^{N_{r}} \ell_{j, \text { ave }} \mathbb{E}\left[\rho_{j}(t)\right] \\
& =T_{\max } \sum_{j=1}^{N_{r}} \ell_{j, \text { ave }} \operatorname{Prob}_{j}^{\text {con }},
\end{aligned}
$$




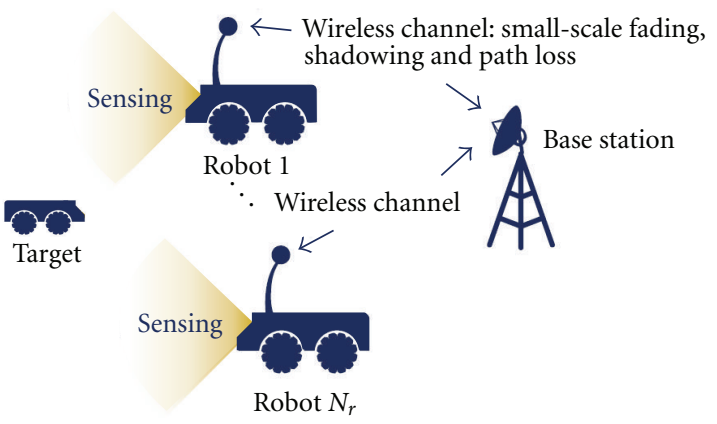

FIGURE 14: Cooperative target tracking over realistic communication links [30].

where $l_{j \text {,ave }}$ is the average information that the $j$ th node collects in this environment (averaged over random positions). In several applications, the robot tries to maintain a fixed distance from the target which maximizes the information collected (sweet spot radius [36]). In such cases, $\boldsymbol{l}_{j}(t)$ is not a function of $t$ anymore and $\ell_{j \text {,ave }}=\ell_{j}(t)$ for any time instant $t$. $\operatorname{Prob}_{j}^{\text {con }} \triangleq \operatorname{Prob}\left\{\mathrm{P}_{r, j}(t)>P_{\mathrm{TH}}\right\}$ is the probability (percentage of the times) that the received power in the transmission of the $j$ th node is above the threshold in the given operation environment. This probability can be calculated using the probabilistic framework of the previous section. We next show how to do this analysis for two different scenarios. Without loss of generality, we assume that all the robots have the same transmit power in this analysis. As such, we drop the index $j$ if the calculation is the same for all the nodes.

First consider the case where the area of operation is small enough, such that the channels can be considered stationary. We will have the following by considering only small-scale fading with a Rayleigh distribution (see Section 3.1):

$$
\operatorname{Prob}^{\text {con }}=\int_{\sqrt{P_{\mathrm{TH}} / P_{T}}}^{\infty} \frac{2 z}{\bar{P}_{z}} \exp \left(\frac{-z^{2}}{\bar{P}_{z}}\right) d z=e^{-P_{\mathrm{TH}} / P_{T} \bar{P}_{z}},
$$

where $P_{T}$ is the TX power (which is taken to be the same for all the nodes) and $\bar{P}_{z}$ represents the average power of the channel (averaged over small-scale fading), as defined in Section 3. Thus, we will have

$$
\ell_{b, \text { total }, \text { ave }}=T_{\max } e^{-P_{\mathrm{TH}} / P_{T} \bar{P}_{z}} \sum_{j=1}^{N_{r}} \ell_{j, \text { ave }} .
$$

Similar expressions can be derived for Rician and Nakagami distributions.

For several robotic applications, however, the area of operation is typically large such that wireless channels can not be considered stationary. If the area and the distance between consecutive channel usages as well are large enough, shadowing and path loss can become the dominant factors. We next characterize Prob ${ }^{\text {con }}$ for such cases, where we can neglect small-scale variations. Using the probabilistic framework of the previous section, we model each received power (in $\mathrm{dB}$ ) as a Gaussian random variable whose average follows the distance-dependent path loss. Due to the space-varying nature of the average, calculating Prob ${ }^{\mathrm{con}}$ is more challenging in this case. Since the distance-dependent average is constant in a given radius from the base station, we calculate Prob $^{\text {con }}$ as follows for the case where the given area can be considered a disk. Consider a narrow ring around the base station between the two disks of radii $R$ and $R+\Delta R$. We have [16]

$$
\begin{aligned}
C_{R, R+\Delta R} & \triangleq \text { area in the ring with received power above } P_{\mathrm{TH}} \\
& \simeq \operatorname{Prob}\left\{P_{r}(R)>P_{\mathrm{TH}}\right\} 2 \pi R \Delta R,
\end{aligned}
$$

where $P_{r}(R)$ is the received power in the transmission from a position at distance $R$ from the base station. Let $R_{\max }$ represent the maximum radius of the area. By integrating $C_{R, R+\Delta R}$ over the whole area, we will have

$$
\begin{aligned}
& \operatorname{Prob}^{\text {con }} \\
& =\frac{2 \pi}{\pi R_{\max }^{2}} \int_{0}^{R_{\max }} R \operatorname{Prob}\left\{P_{r}(R)>P_{\mathrm{TH}}\right\} d R \\
& =\frac{2 \pi}{\pi R_{\max }^{2}} \int_{0}^{R_{\max }} R Q\left(\frac{P_{\mathrm{TH}, \mathrm{dB}}-P_{T, \mathrm{~dB}}-K_{\mathrm{dB}}+10 \gamma \log _{10}(R)}{\sigma_{\mathrm{dB}}}\right) d R \\
& =\frac{2}{R_{\max }^{2}} \int_{0}^{R_{\max }} R Q\left(a+b \ln \frac{R}{R_{\max }}\right) d R \\
& =Q(a)+\exp \left(\frac{2-2 a b}{b^{2}}\right) Q\left(\frac{2-a b}{b}\right),
\end{aligned}
$$

where $a=\left(P_{\mathrm{TH}, \mathrm{dB}}-P_{T, \mathrm{~dB}}-K_{\mathrm{dB}}+10 \gamma \log _{10}\left(R_{\max }\right)\right) / \sigma_{\mathrm{dB}}$, $b=\left(10 \gamma \log _{10}(e)\right) / \sigma_{\mathrm{dB}}, P_{T, \mathrm{~dB}}=10 \log _{10}\left(P_{T}\right), P_{\mathrm{TH}, \mathrm{dB}}=$ $10 \log _{10}\left(P_{\mathrm{TH}}\right)$, and $Q(\alpha)=(1 / \sqrt{2 \pi}) \int_{\alpha}^{\infty} e^{-9^{2} / 2} d \vartheta$ is the $Q-$ function. The last line of (14) is derived after a long but straightforward derivation [16]. Thus we have the following for the average overall received Fisher information at the base station:

$$
\begin{aligned}
& \ell_{b, \text { total ave }} \\
& \quad=T_{\max }\left[Q(a)+\exp \left(\frac{2-2 a b}{b^{2}}\right) Q\left(\frac{2-a b}{b}\right)\right] \sum_{j=1}^{N_{r}} \ell_{j, \text { ave }} .
\end{aligned}
$$

This example shows how the previous channel modeling framework can be used for the probabilistic analysis of robotic networks in realistic communication environments, which becomes considerably important when designing robust cooperative mobile systems.

\section{Optimization of Networked Robotic Systems in the Presence of Realistic Wireless Channels}

In the previous section, we studied how to probabilistically analyze the performance of networked control and robotic 
systems, in the presence of realistic wireless channels. In networked robotic systems, each robot also needs to optimize its motion such that it can gather as much information from the environment as possible, while maintaining connectivity with some of the other nodes or a fixed station. In order to do this, however, a robot needs to (1) have an assessment of the communication link quality in locations it has not yet visited and (2) properly integrate its channel assessment with sensing goals and form communication-aware motion-planning objectives. These two issues make communication-aware motion planning in realistic communication environments a considerably challenging task. In this section, we summarize how the probabilistic channel characterization of Section 3 has recently been utilized for channel learning $[31,37]$ and communication-aware motion planning [30, 32].

5.1. Probabilistic Assessment of Wireless Channels [31, 37]. In this part, we summarize the probabilistic channel assessment framework of $[31,37]$. Consider a team of $N_{r}$ mobile nodes that need to operate in a given workspace $\mathcal{W} \in \mathbb{R}^{2}$ while maintaining their connectivity to a fixed station. Let $P_{(z, \mathrm{~dB})}(q)=10 \log _{10}\left(P_{z}(q)\right)$ denote the channel power in $\mathrm{dB}$, as measured by a node at position $q \in W$. Based on the probabilistic characterization of Section $4, P_{z, \mathrm{~dB}}(q)$ can be expressed as follows:

$$
\begin{aligned}
P_{z, \mathrm{~dB}}(q)= & \widetilde{K}_{\mathrm{dB}}-10 \gamma \log _{10}\left(\left\|q-q_{b}\right\|\right)+P_{z, \mathrm{SH}, \mathrm{ZM}, \mathrm{dB}}(q) \\
& +P_{z, \mathrm{MP}, \mathrm{ZM}, \mathrm{dB}}(q),
\end{aligned}
$$

where $q_{b}=\left(x_{b}, y_{b}\right)$ is the position of the base station, $\widetilde{K}_{\mathrm{dB}}$ and $\gamma$ are path loss parameters, and $P_{z, \mathrm{SH}, \mathrm{ZM}, \mathrm{dB}}(q)$ and $P_{z, \mathrm{MP}, \mathrm{ZM}, \mathrm{dB}}(q)$ are zero-mean random variables representing the effects of shadowing and multipath fading in $\mathrm{dB}$, respectively. Note that $\widetilde{K}_{\mathrm{dB}}$ includes both the average of multipath component in $\mathrm{dB}$, and $K_{\mathrm{dB}}$ (defined in (4)), in order to make $P_{z, \mathrm{MP}, \mathrm{ZM}, \mathrm{dB}}(q)$ zero mean.

As was proved in $[31,37]$, based on the measurements available to the $j$ th node at time $t$ and assuming lognormal shadowing and multipath fading, the assessment of the channel (in $\mathrm{dB}$ ) at an unvisited position $q$ is given by a Gaussian distribution with mean $\hat{P}_{z, \mathrm{~dB}, j, t}(q)=\mathbb{E}\left\{P_{z, \mathrm{~dB}}(q) \mid Y_{j, t}\right\}$ and variance $\delta_{j, t}^{2}(q)=\mathbb{E}\left\{\left(P_{z, \mathrm{~dB}}(q)-\widehat{P}_{z, \mathrm{~dB}, j, t}(q)\right)^{2} \mid Y_{j, t}\right\}$, where $Y_{j, t}$ is the stacked vector of channel power measurements taken at the set of positions $\mathcal{Q}_{j, t}=\left\{q_{1}, \ldots, q_{N_{c}}\right\}$. We then have

$$
\begin{aligned}
\hat{P}_{z, \mathrm{~dB}, j, t}(q)= & \psi^{T}(q) \hat{\omega}_{j, t}+\hat{\phi}_{j, t}^{T}(q) \hat{U}_{j, t}^{-1}\left(Y_{j, t}-\Psi_{j, t} \hat{\omega}_{j, t}\right) \\
\delta_{j, t}^{2}(q)= & \hat{\sigma}_{\mathrm{dB}, j, t}^{2}+\widehat{\omega}_{\mathrm{dB}, j, t}^{2}-\hat{\phi}_{j, t}^{T}(q) \hat{U}_{j, t}^{-1} \hat{\phi}_{j, t}(q) \\
& +\left[\psi(q)-\Psi_{j, t}^{T} \hat{U}_{j, t}^{-1} \hat{\phi}_{j, t}(q)\right]^{T} \Delta_{\varpi, j, t} \\
& \times\left[\psi(q)-\Psi_{j, t}^{T} \hat{U}_{j, t}^{-1} \hat{\phi}_{j, t}(q)\right],
\end{aligned}
$$

where $\hat{\rho}_{\mathrm{dB}, j, t}, \hat{X}_{c, j, t}, \widehat{\omega}_{\mathrm{dB}, j, t}$, and $\hat{\omega}_{j, t}$ denote the estimated values of $\sigma_{\mathrm{dB}}, X_{c}, \omega_{\mathrm{dB}}$, and $\emptyset=\left[\widetilde{K}_{\mathrm{dB}} \gamma\right]^{T}$, based on the available measurements, $\psi(q)=\left[1-10 \log \left(\left\|q-q_{b}\right\|\right)\right]^{T}, \hat{U}_{j, t}=\hat{S}_{j, t}+$ $\hat{\omega}_{\mathrm{dB}, j, t}^{2} I_{N_{c}}$, and $\hat{S}_{j, t}$ denotes the corresponding estimation of the covariance matrix of the shadowing component [31, 37], with $\sigma_{\mathrm{dB}}$ and $X_{c}$ replaced by $\hat{\sigma}_{\mathrm{dB}, j, t}$ and $\hat{X}_{c, j, t}$, respectively. Furthermore, $\Delta_{\varrho, j, t}$ is the estimation error covariance of the path loss parameters, (we skip the details of how to estimate the underlying parameters and refer the readers to $[31,37]) \Psi_{j, t}=\left[1_{N_{c}}-D_{j, t}\right]$, with $1_{N_{c}}$ denoting the $N_{c}$-dimensional vector of all ones and $D_{j, t}=\left[10 \log _{10}\left(\left\|q_{1}-q_{b}\right\|\right) \cdots 10 \log _{10}\left(\left\|q_{N_{c}}-q_{b}\right\|\right)\right]^{T}$, and $\hat{\phi}_{j, t}(q)=\left[\hat{\sigma}_{\mathrm{dB}, j, t}^{2} e^{-\left\|q-q_{1}\right\| / \hat{X}_{c, j, t}} \cdots \hat{\sigma}_{\mathrm{dB}, j, t}^{2} e^{-\left\|q-q_{N_{c}}\right\| / \hat{X}_{c, j, t}}\right]^{T}$.

5.2. Communication-Aware Target Tracking in Robotic Networks [30, 32]. Next, we briefly summarize the communication-aware design and motion planning of $[30,32]$, to show how the channel prediction framework of the previous part can be used for the communication-aware optimization of robotic networks. Consider the target tracking scenario of Section 4 as an example. We found the overall instantaneous Fisher information at the base station to be as follows:

$$
\ell_{b}(t)=\Pi_{b}^{-1}(t)=\sum_{j=1}^{N_{r}} \rho_{j}(t) \ell_{j}(t) .
$$

By averaging only over the distribution of the channel at time instant $t$ (and not over trajectories like the previous section), we obtain

$$
\ell_{b, \text { ave }}(t)=\sum_{j=1}^{N_{r}} \ell_{j}(t) \mathbb{E}\left[\rho_{j}(t)\right]=\sum_{j=1}^{N_{r}} \ell_{j}(t) \operatorname{Prob}_{j}^{\text {con }}(t),
$$

where $\operatorname{Prob}_{j}^{\text {con }}(t)=\operatorname{Prob}\left\{\mathrm{P}_{r, j}(t)>P_{\mathrm{TH}}\right\}$ is the probability that the received power in the transmission of the $j$ th node is above the threshold at time instant $t$. In order for the $j$ th node to maximize its contribution to the Fisher information at the base station, it has to plan its motion such that $\ell_{j}(t) \operatorname{Prob}_{j}^{\text {con }}(t)$ is maximized. This requires predicting $\operatorname{Prob}_{j}^{\text {con }}(t)$ at locations that it has not yet visited. Our aforementioned prediction framework can be utilized towards this prediction as follows:

$$
\operatorname{Prob}_{j}^{\text {con }}(t)=Q\left(\frac{P_{\mathrm{TH}} / P_{T}-\hat{P}_{z, \mathrm{~dB}, j, t}\left(q_{r, j}(t)\right)}{\delta_{j, t}\left(q_{r, j}(t)\right)}\right),
$$

where $q_{r, j}(t)$ is the position of the $j$ th node at time $t$ and $P_{T}$ is the TX power. Then the $j$ th node can optimize its motion as follows [30, 32]:

$$
\begin{aligned}
u_{j, r}^{*}(t)= & \underset{u_{j, r}(t)}{\arg \max } Q\left(\frac{P_{\mathrm{TH}} / P_{T}-\widehat{P}_{z, \mathrm{~dB}, j, t}\left(q_{r, j}(t+1)\right)}{\delta_{j, t}\left(q_{r, j}(t+1)\right)}\right) \\
& \times \ell_{j}(t+1) \\
\text { s.t. } q_{r, j}(t+1)= & \Upsilon_{j}\left(q_{r, j}(t), u_{j, r}(t)\right), \quad u_{j, r}(t) \in \mathcal{U},
\end{aligned}
$$

where $q_{r, j}(t+1)=\Upsilon_{j}\left(q_{r, j}(t), u_{j, r}(t)\right)$ denotes the dynamics of the $j$ th node, with $u_{j, r}(t)$ denoting its control input, and $U$ is the set of admissible control signals. 


\section{Developing a Probabilistic Channel Generation Environment for Networked Robotic Applications}

Developing a realistic channel simulation environment is considerably important for testing the performance of any proposed networked robotic strategy. If all the information about object positions, geometry, and dielectric properties is available, ray tracing methods could be used to find a spatial map of the received signal strength in the area of interest. A ray tracer [38] follows all or some of the reflected, diffracted or scattered multipaths in the environment. There are several software packages, such as Wireless InSite [39] and Motorola's WLAN Planner and Site Scanner, that are aimed at generating a map of the received signal strength based on ray tracing. While it is possible to use such software for evaluating the performance of a robotic network in a certain environment, assessing the exact coefficients associated with the dielectric properties of the objects could be challenging. Furthermore, such commercial software packages are typically considerably expensive. Finally, unlike probabilistic characterizations of the previous section, ray tracing approaches are not suitable for mathematical analysis since they simply generate a received signal map for a specific environment.

The characterization of the previous sections can be used towards developing a probabilistic channel simulator. A probabilistic simulator is, in particular, suitable for those cases where the information of the environment, in terms of dielectric/magnetic properties and/or geometries of the obstacles, is not entirely known (which is the case most of the time). Then, channel can be generated as a two-dimensional spatial function with the three major dynamics, that is, smallscale fading, shadowing, and path loss while ensuring the desired spatial correlation. In this section, we show how such a probabilistic channel simulator can be developed.

As discussed earlier, the received signal power, $P_{r}(x, y)$, at position $(x, y) \in \mathbb{R}^{2}$ consists of the following three components.

(i) Path Loss Component $\left(P_{r, \mathrm{PL}}(x, y)\right)$ : The distancedependent path loss component of the received signal power can be expressed as follows in $\mathrm{dB}$ :

$$
\begin{aligned}
& P_{r, \mathrm{PL}, \mathrm{dB}}(x, y) \\
& =10 \log _{10}\left(P_{r, \mathrm{PL}}(x, y)\right) \\
& =\underbrace{P_{T, \mathrm{~dB}}}_{\text {TX power }}+\underbrace{K_{\mathrm{dB}}-10 \gamma \log _{10}\left(\sqrt{\left(x-x_{b}\right)^{2}+\left(y-y_{b}\right)^{2}}\right)}_{\text {path } \operatorname{loss}\left(\mu_{\mathrm{dB}}(x, y) \leq 0\right)},
\end{aligned}
$$

where $\left(x_{b}, y_{b}\right) \in \mathbb{R}^{2}$ is the position of the base station.

(ii) Shadowing Component $\left(P_{r, \mathrm{SH}, \mathrm{ZM}}(x, y)\right)$ : Based on the lognormal distribution of the shadowing component, we have $P_{r, \mathrm{SH}, \mathrm{ZM}, \mathrm{dB}}(x, y)=10 \log _{10}\left(P_{r, \mathrm{SH}, \mathrm{ZM}}(x, y)\right)$ as a 2D zero-mean Gaussian random process with the following Autocovariance Function (ACF):

$$
\begin{aligned}
& A_{\mathrm{cov}, P_{r, \mathrm{SH}, \mathrm{ZM}, \mathrm{dB}}}(\Delta x, \Delta y) \\
& \quad=\mathbb{E}\left[P_{r, \mathrm{SH}, \mathrm{ZM}, \mathrm{dB}}(x+\Delta x, y+\Delta y) P_{r, \mathrm{SH}, \mathrm{ZM}, \mathrm{dB}}(x, y)\right] \\
& \quad=\sigma_{\mathrm{dB}}^{2} e^{-\sqrt{\Delta x^{2}+\Delta y^{2}} / X_{c}},
\end{aligned}
$$

where $\sigma_{\mathrm{dB}}^{2}$ is the variance of the shadowing component in $\mathrm{dB}$ and $X_{c}$ is the decorrelation distance as defined in the previous section. It can be easily shown that the 2D Fourier transform of $A_{\mathrm{cov}, P_{r, \mathrm{SH}, \mathrm{ZM}, \mathrm{dB}}}(\Delta x, \Delta y)$ results in the following Power Spectral Density (PSD) [40]:

$$
\Phi_{P_{r, \mathrm{SH}, \mathrm{ZM}, \mathrm{dB}}}\left(f_{x}, f_{y}\right)=\frac{2 \pi X_{c}^{2} \sigma_{\mathrm{dB}}^{2}}{\left[1+4 \pi^{2} X_{c}^{2}\left(f_{x}^{2}+f_{y}^{2}\right)\right]^{3 / 2}}
$$

(iii) Small-Scale (Multipath) Fading Component $\left(P_{r, \mathrm{MPNORM}}(x, y)\right)$ : Let $h(x, y)$ represent the baseband equivalent channel at position $(x, y) \in \mathbb{R}^{2}$, as introduced in Section 3. Let $h_{\mathrm{NORM}}(x, y)$ denote the normalized version of $h(x, y)$, which has a unit power. $h_{\mathrm{NORM}}(x, y)$ can be described as a function of its in-phase and quadrature components as follows: $h_{\mathrm{NORM}}(x, y)=h_{I, \mathrm{NORM}}(x, y)+j h_{\mathrm{Q}, \mathrm{NORM}}(x, y)$, where $h_{I, \mathrm{NORM}}(x, y)$ and $h_{Q, \mathrm{NORM}}(x, y)$ are the in-phase and quadrature parts, respectively [16]. Then, we have $P_{r, \mathrm{MP}, \mathrm{NORM}}(x, y) \triangleq\left|h_{\mathrm{NORM}}(x, y)\right|^{2}=h_{I, \mathrm{NORM}}^{2}(x, y)+$ $h_{Q, N O R M}^{2}(x, y)$. For Rayleigh-distributed small-scale fading, (Rician small-scale fading can be similarly characterized and simulated by adding a constant to the in-phase or quadrature part) $h_{I, \mathrm{NORM}}(x, y)$ and $h_{Q, N O R M}(x, y)$ are zero-mean Gaussian processes with the power of 0.5 . As discussed earlier, one possible form for the spatial covariance of the in-phase or quadrature components of the small-scale variations is given by the Jakes model as follows $[8,16]$ :

$$
\begin{aligned}
& A_{\mathrm{COV}, P_{r, \mathrm{MP}, \mathrm{NORM}}}(\Delta x, \Delta y) \\
& =\mathbb{E}\left[h_{I, \mathrm{NORM}}(x+\Delta x, y+\Delta y) h_{I, \mathrm{NORM}}(x, y)\right] \\
& =\mathbb{E}\left[h_{\mathrm{Q}, \mathrm{NORM}}(x+\Delta x, y+\Delta y) h_{\mathrm{Q}, \mathrm{NORM}}(x, y)\right] \\
& =\frac{1}{2} J_{0}\left(2 \pi \frac{\sqrt{\Delta x^{2}+\Delta y^{2}}}{\lambda}\right),
\end{aligned}
$$

where $J_{0}(\cdot)$ is the zeroth-order Bessel function and $\lambda$ is the wavelength of operation. This model is valid for the cases where we have uniform angle of arrivals and isotropic antennas. 


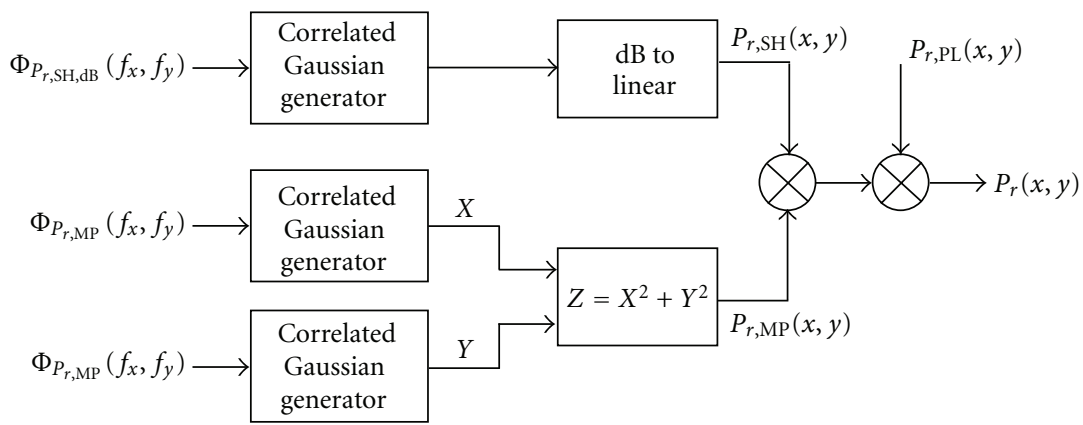

FIGURE 15: Block diagram of the two-dimensional probabilistic channel simulator for networked robotic applications.

The PSD of the in-phase or quadrature component is then given by the following, which can be calculated numerically:

$$
\begin{aligned}
& \Phi_{P_{r, \mathrm{MP}, \mathrm{NORM}}}\left(f_{x}, f_{y}\right) \\
& =\int_{-\infty}^{\infty} \int_{-\infty}^{\infty} \frac{1}{2} J_{0}\left(2 \pi \frac{\sqrt{\Delta x^{2}+\Delta y^{2}}}{\lambda}\right) e^{-j 2 \pi\left(f_{x} \Delta x+f_{y} \Delta y\right)} d \Delta x d \Delta y \\
& =\frac{1}{2} \int_{0}^{2 \pi} \int_{0}^{\infty} J_{0}\left(2 \pi \frac{R}{\lambda}\right) e^{-j 2 \pi R\left(f_{x} \cos (\theta)+f_{y} \sin (\theta)\right)} R d R d \theta .
\end{aligned}
$$

Therefore, in order to generate the small-scale component of the received power, two independent 2D zero-mean Gaussian random processes (with ACF given by (25)) should be generated for the in-phase $\left(h_{I, \mathrm{NORM}}(x, y)\right)$ and quadrature $\left(h_{Q, N O R M}(x, y)\right)$ parts. Then the small-scale component can be generated using $P_{r, \mathrm{MPNORM}}(x, y)=h_{I, \mathrm{NORM}}^{2}(x, y)+$ $h_{\text {Q,NORM }}^{2}(x, y)$.

It can be seen that in order to generate the underlying channel dynamics, 2D Gaussian processes should be generated with appropriate correlation properties. Next, we discuss two different approaches for generating a $2 \mathrm{D}$ correlated Gaussian process. While we provided examples of the most common correlation functions in (23) and (25), any correlation function can be generated in what follows. Once all the three dynamics of the channel are generated, the overall received signal power can be simulated by $P_{r}(x, y)=$ $P_{r, \mathrm{PL}}(x, y) P_{r, \mathrm{SH}, \mathrm{ZM}}(x, y) P_{r, \mathrm{MP}, \mathrm{NORM}}(x, y)$. Figure 15 shows a block diagram of the probabilistic channel generation process.

6.1. Generation of a Correlated 2D Gaussian Process. There exist a number of techniques for generating a correlated Gaussian random process with an arbitrary ACF. Here, we briefly discuss two approaches, which we have used in our own simulation environment.

Filtering-Based Approach [41]. The most intuitive way of generating a correlated Gaussian process is to generate 2D uncorrelated Gaussian variables and pass them through a properly designed filter, whose transfer function is the square root of the desired power spectral density. For instance, in order to generate the in-phase part of the small-scale fading with the ACF of (25), a 2D i.i.d. zero-mean Gaussian random variable with unit variance is passed through a bidirectional filter with the following transfer function:

$$
G\left(f_{x}, f_{y}\right)=\sqrt{\Phi_{P_{r, \mathrm{MPNORM}}}\left(f_{x}, f_{y}\right)} .
$$

The quadrature part can be similarly generated. Note that the resulting small-scale component will have a unit power since $\int_{-\infty}^{\infty} \int_{-\infty}^{\infty}\left|G\left(f_{x}, f_{y}\right)\right|^{2} d f_{x} d f_{y}=1 / 2$.

(i) The filtering approach provides a simple way of generating a correlated Gaussian process. However, it can result in high computational complexity (or poor performance depending on the given computation budget) when the spatial correlation increases. For instance, while it may be suitable for generating correlated small-scale fading, other approaches may be more efficient for generating the correlated shadowing component, as we discuss next.

(ii) Sum-of-Sinusoids-(SOS-) Based Approach [40]. An alternative method for generating a correlated Gaussian process is proposed in [40]. Here, we briefly discuss this approach. A Gaussian random process can be efficiently approximated by the sum of a finite number of sinusoids with random phases and properly selected frequencies and amplitudes [40]. For instance, consider the shadowing component in $\mathrm{dB}$. It can be written as follows:

$$
P_{r, \mathrm{SH}, \mathrm{ZM}, \mathrm{dB}}(x, y)=\sum_{j=1}^{N_{s}} c_{j} \cos \left[2 \pi\left(f_{x, j} x+f_{y, j} y\right)+\theta_{j}\right] \text {, }
$$

where $N_{s}$ is the number of sinusoids, $\left\{f_{x, j}\right\}_{j=1}^{N_{s}}$ and $\left\{f_{y, j}\right\}_{j=1}^{N_{s}}$ are discrete spatial frequencies that can be either deterministic (uniform or nonuniform) or random, $\left\{\theta_{j}\right\}_{j=1}^{N_{s}}$ are randomly distributed over $[0,2 \pi)$, and constants $\left\{c_{j}\right\}_{j=1}^{N_{s}}$ are calculated based on the PSD of the shadowing component (e.g., in (24)). As an example, we briefly explain how $\left\{f_{x, j}\right\}_{j=1}^{N_{s}},\left\{f_{y, j}\right\}_{j=1}^{N_{s}}$ and $\left\{c_{j}\right\}_{j=1}^{N_{s}}$ can be selected for the case with non-uniform but deterministic sampling of the spatial frequencies. Readers are referred to [40] for more details. 


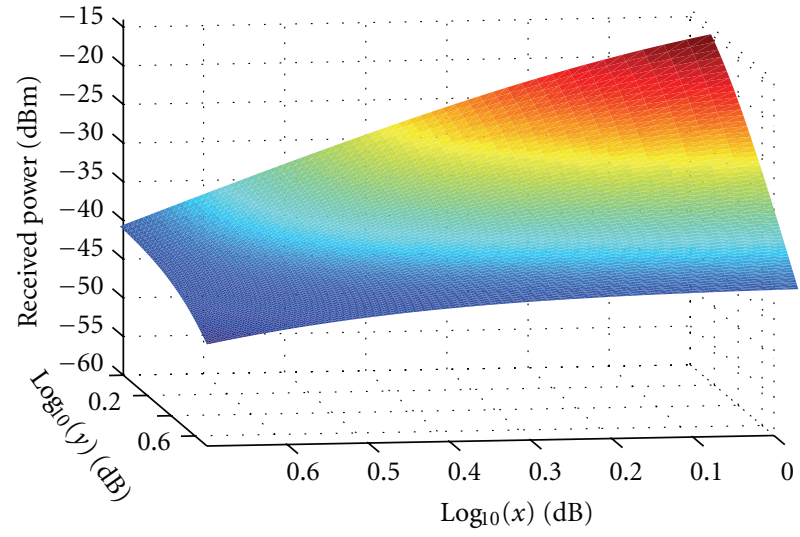

(a)

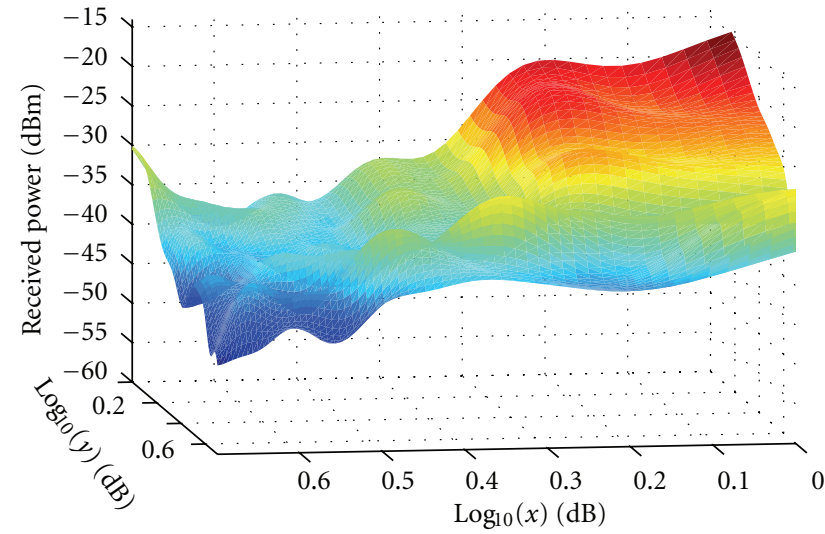

(b)

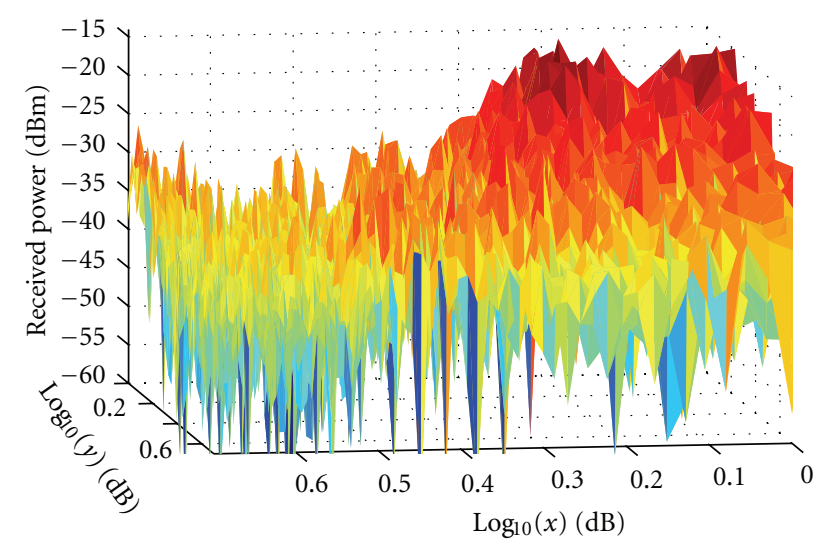

(c)

FIGURE 16: An example of a channel generated using the probabilistic characterization of the previous section. (a) Channel with path loss only, (b) channel with path loss and shadowing, and (c) channel with path loss, shadowing, and small-scale fading. Transmitter is in the top right corner.

As can be seen from (24), the PSD of shadowing is circularly symmetric. Thus, by defining $f_{x}=f_{r} \cos (\varphi)$ and $f_{y}=f_{r} \sin (\varphi)$, the $1 \mathrm{D}$ equivalent PSD is given by $\Phi_{P_{r, S H, Z M, d B}}\left(f_{r}\right)=2 \pi X_{c}^{2} \sigma_{\mathrm{dB}}^{2} /\left[1+4 \pi^{2} X_{c}^{2} f_{r}^{2}\right]^{3 / 2}$. Define the $\mathcal{E}$ $\mathrm{dB}$ cutoff frequency $\left(f_{r, c}\right)$ as the radial frequency for which $\Phi_{P_{r, \mathrm{SH}, \mathrm{ZM}, \mathrm{dB}}}\left(f_{r, c}\right) / \Phi_{P_{r, \mathrm{SH}, \mathrm{ZM}, \mathrm{dB}}}(0)=10^{-\varepsilon / 10}$. This gives $f_{r, c}=$ $1 /\left(2 \pi X_{c}\right) \sqrt{10^{2 \varepsilon / 30}-1}$. Define $M_{s}$ such that $N_{s}=2 M_{s}^{2}$. We first pick $M_{s}$ radial frequencies $\left\{f_{r, k}\right\}_{k=1}^{M_{s}}$ such that

$$
\begin{aligned}
& \int_{0}^{2 \pi} \int_{f_{r, k-1}}^{f_{r, k}} \Phi_{P_{r, \mathrm{SH}, \mathrm{ZM}, \mathrm{dB}}}\left(f_{r}\right) f_{r} d f_{r} d \varphi \\
& \quad=\frac{1}{M_{s}} \int_{0}^{2 \pi} \int_{0}^{f_{r, c}} \Phi_{P_{r, \mathrm{SH}, \mathrm{ZM}, \mathrm{dB}}}\left(f_{r}\right) f_{r} d f_{r} d \varphi,
\end{aligned}
$$

starting from $f_{r, 0}=0$. By picking up $2 M_{s}$ angles in $(-\pi / 2, \pi / 2)$ using $\left\{\varphi_{l}=\pi\left(2 l-2 M_{s}+1\right) / 4 M_{s}\right\}_{l=0}^{2 M_{s}-1}$, the $N_{s}$ sampling frequencies are given by $f_{x, j}=f_{r, k} \cos \left(\varphi_{l}\right)$ and $f_{y, j}=f_{r, k} \sin \left(\varphi_{l}\right)$, where $k=\left\lfloor(j-1) / 2 M_{s}\right\rfloor+1$ and $l=$ $j \bmod 2 M_{s}$. Since the power in the area between any two radial frequencies $f_{r, k-1}$ and $f_{r, k}$ for $k=1, \ldots, M_{s}$ is the same, we can choose $c_{j}=\sigma_{\mathrm{dB}} \sqrt{2 / N_{s}}$. It can be shown that by using a moderately large value for $N_{s}$, the average mean square error between the desired and generated ACF is very small [40].
Figure 16 shows an example of a two-dimensional channel generated by using the characterization of the previous sections and the probabilistic simulator of this section. The figure shows the three different dynamics of the channel. For instance, the Figure 16(a) shows only the distancedependent path loss whereas Figure 16(b) shows the shadowing component added. Finally, Figure 16(c) shows the channel with all the three scales. In this example, the covariance functions for small scale and shadowing are taken to be Bessel and exponential, respectively, as discussed in the previous section. The autocovariance function of the simulated shadowing component is shown in Figure 17(a). The figure shows a slice of the autocovariance along a fixed position in the $x$ axis. The desired autocovariance corresponds to an exponentially decaying function given by (23). As can be seen, the two curves match well. Similarly, Figure 17(b) shows a slice of the desired and simulated normalized small-scale autocorrelation functions. A good match can be seen.

6.2. Case of Partially Known Environment. In robotic applications, the positions and geometry of some of the objects are learned for navigation purposes. Such knowledge can also be incorporated in the channel simulator such that 


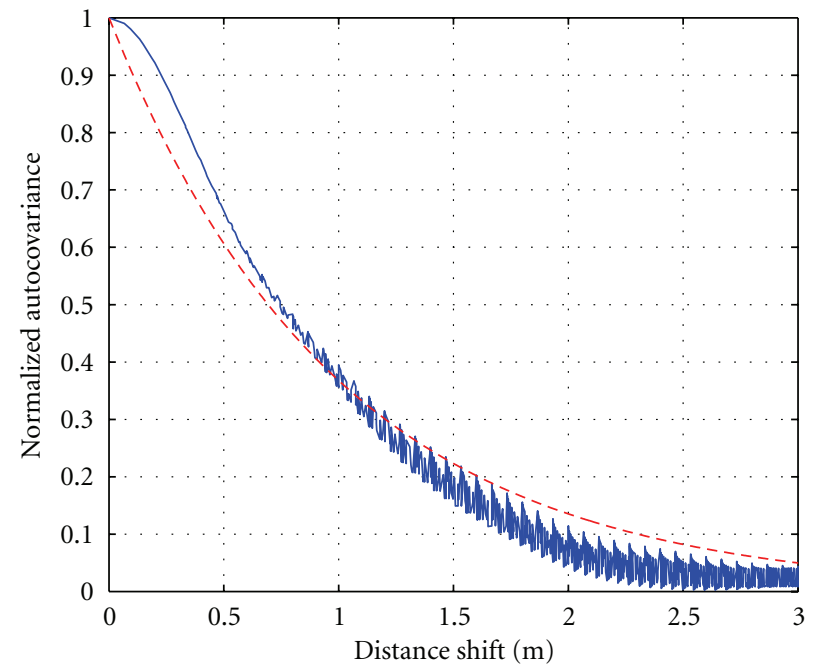

— Autocovariance of shadowing for simulated channel
- - Desired autocovariance function

(a)

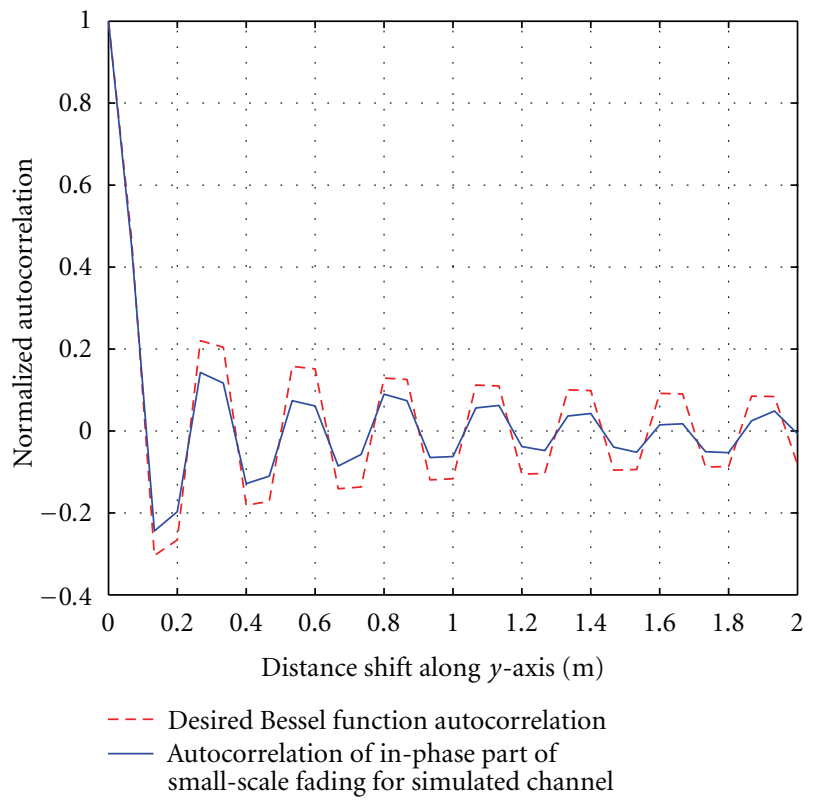

(b)

FIGURE 17: (a) A slice of the normalized autocovariance function of shadowing for the simulated two-dimensional channel of Figure 16 and its exponential match. (b) A slice of the autocorrelation function of the in-phase part of small-scale fading and its Bessel function match. It can be seen that our simulated channel has the desired correlation properties.

the generated channel matches the known environmental specifications better. For instance, consider the case where the position and geometry of the obstacles are known on the line from position $(x, y) \in \mathbb{R}^{2}$ to the transmitter. Then, the received signal power in $\mathrm{dB}, P_{r, \mathrm{~dB}}(x, y)$, at position $(x, y)$, can be approximated as follows by following the LOS path and considering only shadow fading and path loss:

$$
\begin{aligned}
P_{r, \mathrm{~dB}} & (x, y) \\
\approx & \underbrace{P_{T, \mathrm{~dB}}}_{\text {TX power }}+\underbrace{K_{\mathrm{dB}}-10 \gamma \log _{10}\left(\sqrt{\left(x-x_{b}\right)^{2}+\left(y-y_{b}\right)^{2}}\right)}_{\text {path } \operatorname{loss}\left(\mu_{\mathrm{dB}}(x, y) \leq 0\right)} \\
& -\zeta \underbrace{\zeta \sum_{j} \beta_{\text {los }, j} l_{\text {los }, j},}_{\text {shadowing }}
\end{aligned}
$$

where $\zeta=10 / \ln (10)$, path loss parameters are as defined earlier, and $\beta_{\mathrm{los}, j}$ and $l_{\mathrm{los}, j}$ denote the approximated decay rate of the LOS path and its traveled distance in the $j$ th obstacle on its path, respectively. It should be noted that implementing (30) still requires approximating $\beta_{\mathrm{los}, j}$ for all $j$ s. In practice, this approximation can only be done very roughly. Furthermore, we still have to simulate small-scale fading. As such, using only (30) does not suffice for channel generation. Instead, we can combine partial environmentspecific knowledge with probabilistic components of the previous section in order to generate a more realistic channel. For instance, a small-scale fading variable can be added to the received power generated from (30). Figure 18 shows simulated binary maps of the received signal power where black areas indicate regions with the received signal strength below an acceptable threshold while white areas denote otherwise. Figure 18(a) shows an example of a channel generated based on knowing the positions of the obstacles and considering only shadowing and path loss, as indicated by (30), and for the marked transmitter location. Figure 18(b) shows the same map after adding a Riciandistributed small-scale fading to it, which becomes more realistic.

\section{Conclusions}

The goal of this paper was to provide a reference for researchers in robotics and control that are interested in the realistic characterization of wireless links. By utilizing the knowledge available in wireless communication literature, we provided a comprehensive overview of the key characteristics of wireless channels: small-scale fading, shadowing, and path loss, for networked robotic and control operations. We furthermore developed a robotic testbed in order to confirm these mathematical models. We then summarized some of the recent results on how to use this probabilistic framework for the analysis and optimization of networked robotic systems. Finally, we showed how to develop a realistic yet simple channel simulator for the verification of cooperative robotic operations in realistic communication environments. 


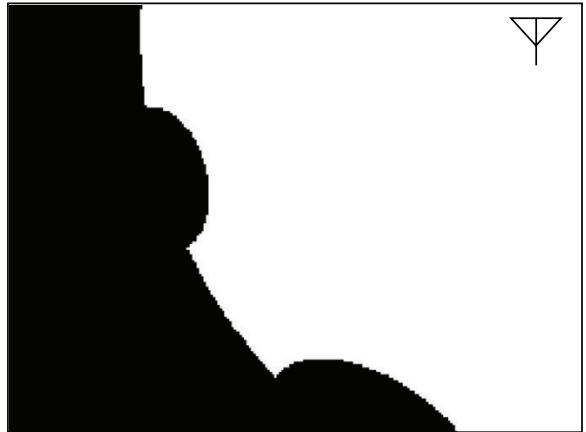

(a)

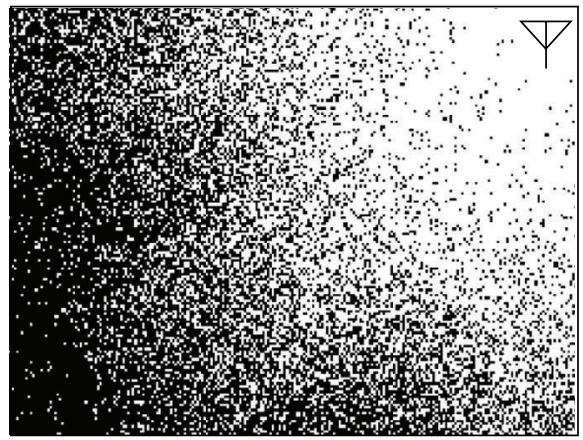

(b)

FIGURE 18: Examples of a binary channel (white areas indicate that channel quality is above an acceptable threshold whereas black areas denote otherwise) - (a) channel generated based on knowing the positions of the obstacles and considering only shadowing and path loss and (b) the same channel after adding Rician-distributed fading.

\section{Acknowledgments}

This work is supported in part by NSF IIS Award no. 0812338 and ARO CTA MAST Project no. W911NF-08-2-0004. A small part of this work is presented in the International Conference on Intelligent RObots and Systems (IROS), 2009 [42].

\section{References}

[1] R. Olfati-Saber and R. M. Murray, "Distributed structural stabilization and tracking for formations of dynamic multiagents," in Proceedings of the 41st IEEE Conference on Decision and Control, pp. 209-215, Las Vegas, Nev, USA, December 2002.

[2] H. G. Tanner, A. Jadbabaie, and G. J. Pappas, "Stable flocking of mobile Agents, part I: fixed topology," in Proceedings of the 42nd IEEE Conference on Decision and Control, pp. 2010-2015, Maui, Hawaii, USA, December 2003.

[3] S. Tatikonda, Control under communication constraints, Ph.D. dissertation, MIT, 2000.

[4] B. Grocholsky, J. Keller, V. Kumar, and G. Pappas, "Cooperative air and ground surveillance," IEEE Robotics and Automation Magazine, vol. 13, no. 3, pp. 16-26, 2006.

[5] A. Rahmani, M. Ji, M. Mesbahi, and M. Egerstedt, "Controllability of multi-agent systems from a graph-theoretic perspective," SIAM Journal on Control and Optimization, vol. 48, no. 1, pp. 162-186, 2009.
[6] G. Notarstefano, K. Savla, F. Bullo, and A. Jadbabaie, "Maintaining limited-range connectivity among second-order agents," in Proceedings of the American Control Conference, pp. 2124-2129, June 2006.

[7] J. M. Esposito and T. W. Dunbar, "Maintaining wireless connectivity constraints for swarms in the presence of obstacles," in Proceedings of the IEEE International Conference on Robotics and Automation (ICRA '06), pp. 946-951, May 2006.

[8] W. C. Jakes, Microwave Mobile Communications, Wiley-IEEE Press, New York, NY, USA, 1994.

[9] R. Davies, M. Bensebti, M. A. Beach, and J. P. McGeehan, "Wireless propagation measurements in indoor multipath environments at $1.7 \mathrm{GHz}$ and $60 \mathrm{GHz}$ for small cell systems," in Proceedings of the 41st IEEE Vehicular Technology Conference, Gateway to the Future Technology in Motion, pp. 589-593, May 1991.

[10] T. S. Rappaport and S. Y. Seidel, "Multipath propagation models for in-building communications," in Proceedings of the 5th International Conference on Mobile Radio and Personal Communications, pp. 69-74, 1989.

[11] H. Nikookar and H. Hashemi, "Statistical modeling of signal amplitude fading of indoor radio propagation channels," in Proceedings of the 2nd International Conference on Universal Personal Communications, vol. 1, pp. 84-88, 1993.

[12] D. C. Cox, " $910 \mathrm{MHz}$ urban mobile radio propagation: multipath characteristics in New York city," IEEE Transactions on Vehicular Technology, vol. 22, no. 4, pp. 104-110, 1973.

[13] D. C. Cox, "Delay doppler characteristics of multipath propagation at $910 \mathrm{MHz}$ in a suburban mobile radio environment," IEEE Transactions on Antennas and Propagation, vol. 20, no. 5, pp. 625-635, 1972.

[14] H. Hashemi, "Indoor radio propagation channel," Proceedings of the IEEE, vol. 81, no. 7, pp. 943-968, 1993.

[15] T. S. Rappaport, Wireless Communications: Principles and Practice, Prentice-Hall, Upper Saddle River, NJ, USA, 2002.

[16] A. Goldsmith, Wireless Communications, Cambridge University Press, New York, NY, USA, 2005.

[17] D. Tse and P. Viswanath, Fundamentals of Wireless Communication, Cambridge University Press, New York, NY, USA, 2005.

[18] D. Porrat and D. C. Cox, "UHF propagation in indoor hallways," IEEE Transactions on Wireless Communications, vol. 3, no. 4, pp. 1188-1198, 2004.

[19] J. Tsao, D. Porrat, and D. Tse, "Prediction and modeling for the time-evolving ultra-wideband channel," IEEE Journal on Selected Topics in Signal Processing, vol. 1, no. 3, pp. 340-356, 2007.

[20] P. Kyritsi, D. C. Cox, R. A. Valenzuela, and P. W. Wolniansky, "Correlation analysis based on MIMO channel measurements in an indoor environment," IEEE Journal on Selected Areas in Communications, vol. 21, no. 5, pp. 713-720, 2003.

[21] J. Ling, D. Chizhik, P. Wolinansky, R. A. Valenzuela, N. Costa, and K. Huber, "MIMO measurement in Manhattan," in Proceedings of the 13th IEEE International Symposium on Personal, Indoor and Mobile Radio Communications, vol. 4, pp. 1631-1635, 2002.

[22] W. M. Smith, Urban propagation modeling for wireless systems, Ph.D. dissertation, Stanford University, 2004.

[23] Y. Lustmann and D. Porrat, "Indoor channel spectral statistics, K-factor and reverberation distance," IEEE Transactions on Antennas and Propagation, vol. 58, no. 11, Article ID 5559353, pp. 3685-3692, 2010. 
[24] MobileRobots Inc., 2009, http://www.mobilerobots.com.

[25] Laird Technologies, 2010, http://www.lairdtech.com/Products/Antennas-and-Reception-Solutions/.

[26] N. Nakagami, "The m-distribution, a general formula for intensity distribution of rapid fading," in Statistical Methods in Radio Wave Propagation, W. G. Hoffman, Ed., Pergamon, Oxford, UK, 1960.

[27] H. Hashemi, "A study of temporal and spatial variations of the indoor radio propagation channel," in Proceedings of the 5th IEEE International Symposium on Personal, Indoor and Mobile Radio Communications, vol. 1, pp. 127-134, 1994.

[28] V. Erceg, L. J. Greenstein, S. Y. Tjandra et al., "Empirically based path loss model for wireless channels in suburban environments," IEEE Journal on Selected Areas in Communications, vol. 17, no. 7, pp. 1205-1211, 1999.

[29] S. S. Ghassemzadeh, L. J. Greenstein, A. Kavčić, T. Sveinsson, and V. Tarokh, "UWB indoor path loss model for residential and commercial buildings," in Proceedings of the IEEE Vehicular Technology Conference (VTC '03), vol. 5, pp. 3115-3119, October 2003.

[30] A. Ghaffarkhah and Y. Mostofi, "Communication-aware motion planning in mobile networks," IEEE Transactions on Automatic Control, vol. 56, no. 10, pp. 2478-2485, 2011.

[31] Y. Mostofi, M. Malmirchegini, and A. Ghaffarkhah, "Estimation of communication signal strength in robotic networks," in Proceedings of the IEEE International Conference on Robotics and Automation (ICRA '10), pp. 1946-1951, Anchorage, Alaska, USA, May 2010.

[32] A. Ghaffarkhah and Y. Mostofi, "Channel learning and communication-aware motion planning in mobile networks," in Proceedings of the American Control Conference (ACC '10), pp. 5413-5420, Baltimore, Md, USA, July 2010.

[33] M. Gudmundson, "Correlation model for shadow fading in mobile radio systems," Electronics Letters, vol. 27, no. 23, pp. 2145-2146, 1991.

[34] Y. Mostofi, "Decentralized communication-aware motion planning in mobile networks: an information-gain approach," Journal of Intelligent and Robotic Systems, vol. 56, no. 1-2, pp. 233-256, 2009.

[35] H. V. Poor, An Introduction to Signal Detection and Estimation, Springer, New York, NY, USA, 1994.

[36] A. Ghaffarkhah and Y. Mostofi, "Communication-aware navigation functions for robotic networks," in Proceedings of the American Control Conference (ACC '09), pp. 1316-1322, St. Louis, Mo, USA, June 2009.

[37] M. Malmirchegini and Y. Mostofi, "On the spatial predictability of communication channels," IEEE Transactions on Wireless Communications. In press.

[38] C. F. Yang, B. C. Wu, and C. J. Ko, "A ray-tracing method for modeling indoor wave propagation and penetration," IEEE Transactions on Antennas and Propagation, vol. 46, no. 6, pp. 907-919, 1998.

[39] Remcom, 2010, http://www.remcom.com/wireless-insite/.

[40] X. Cai and G. B. Giannakis, "A two-dimensional channel simulation model for shadowing processes," IEEE Transactions on Vehicular Technology, vol. 52, no. 6, pp. 1558-1567, 2003.

[41] S. A. Fechtel, "A novel approach to modeling and efficient simulation of frequency-selective fading radio channels," IEEE Journal on Selected Areas in Communications, vol. 11, no. 3, pp. 422-431, 1993.
[42] Y. Mostofi, A. Gonzalez-Ruiz, A. Gaffarkhah, and D. Li, "Characterization and modeling of wireless channels for networked robotic and control systems-a comprehensive overview," in Proceedings of the IEEE/RSJ International Conference on Intelligent Robots and Systems (IROS '09), pp. 4849-4854, St. Louis, Mo, USA, 2009. 

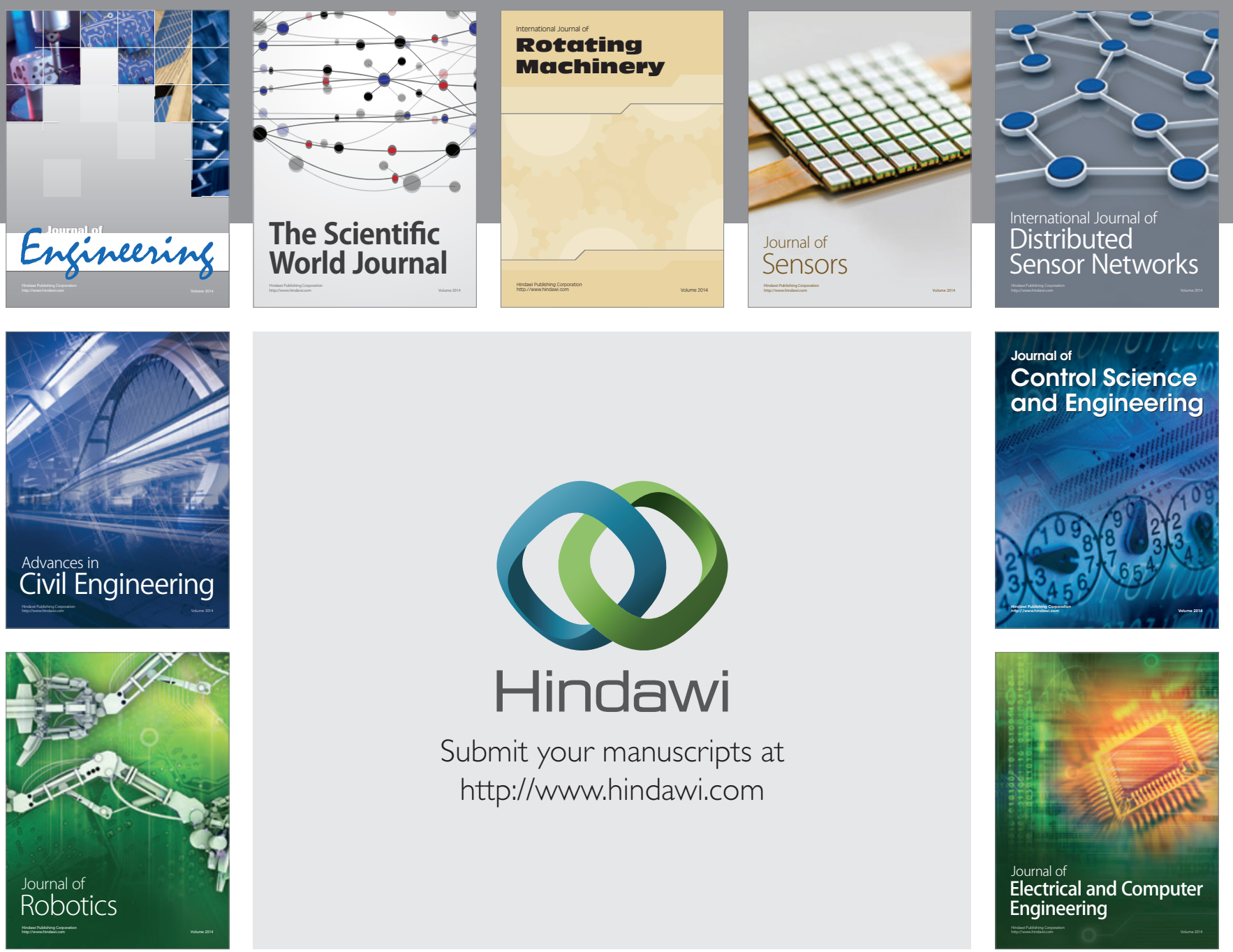

Submit your manuscripts at

http://www.hindawi.com
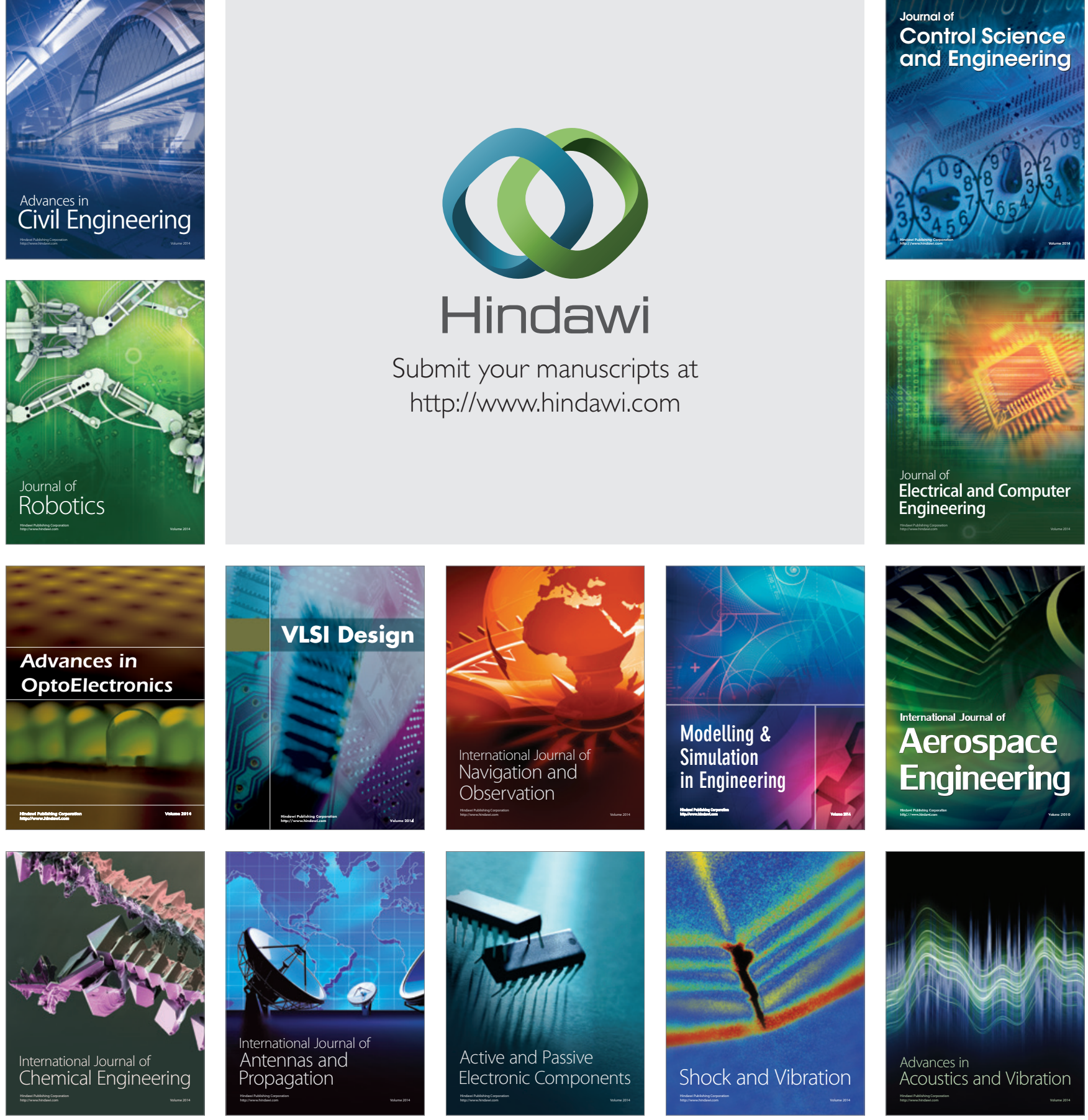